\title{
Properties of isolated disk galaxies ${ }^{\star}$
}

\author{
J. Varela ${ }^{1}$, M. Moles ${ }^{1}$, I. Márquez ${ }^{1}$, G. Galletta $^{2}$, J. Masegosa $^{1}$, and D. Bettoni ${ }^{3}$ \\ 1 Instituto de Astrofísica de Andalucía (C.S.I.C.) Apartado 3004, 18080 Granada, Spain \\ e-mail: jesusv@imaff.cfmac.csic.es; isabel@iaa.es;pepa@iaa.es \\ 2 Dipartimento di Astronomia, Università di Padova, Vicolo dell’Osservatorio 2, 35122 Padova, Italy \\ e-mail: galletta@pd.astro.it \\ 3 Osservatorio Astronomico di Padova, Vicolo dell'Osservatorio 5, 35122 Padova, Italy \\ e-mail: bettoni@pd.astro.it
}

Received 17 November 2003 / Accepted 27 February 2004

\begin{abstract}
We present a new sample of northern isolated galaxies, which are defined by the physical criterion that they were not affected by other galaxies in their evolution during the last few Gyr. To find them we used the logarithmic ratio, $f$, between inner and tidal forces acting upon the candidate galaxy by a possible perturber. The analysis of the distribution of the $f$-values for the galaxies in the Coma cluster lead us to adopt the criterion $f \leq-4.5$ for isolated galaxies. The candidates were chosen from the CfA catalog of galaxies within the volume defined by $c z \leq 5000 \mathrm{~km} \mathrm{~s}^{-1}$, galactic latitude higher than $40^{\circ}$ and declination $\geq-2.5^{\circ}$. The selection of the sample, based on redshift values (when available), magnitudes and sizes of the candidate galaxies and possible perturbers present in the same field is discussed. The final list of selected isolated galaxies includes 203 objects from the initial 1706. The list contains only truly isolated galaxies in the sense defined, but it is by no means complete, since all the galaxies with possible companions under the $f$-criterion but with unknown redshift were discarded. We also selected a sample of perturbed galaxies comprised of all the disk galaxies from the initial list with companions (with known redshift) satisfying $f \geq-2$ and $\Delta(c z) \leq 500 \mathrm{~km} \mathrm{~s}^{-1}$; a total of 130 objects. The statistical comparison of both samples shows significant differences in morphology, sizes, masses, luminosities and color indices. Confirming previous results, we found that late spiral, Sc-type galaxies are, in particular, more frequent among isolated galaxies, whereas Lenticular galaxies are more abundant among perturbed galaxies. Isolated systems appear to be smaller, less luminous and bluer than interacting objects. We also found that bars are twice as frequent among perturbed galaxies compared to isolated galaxies, in particular for early Spirals and Lenticulars. The perturbed galaxies have higher $L_{\mathrm{FIR}} / L_{\mathrm{B}}$ and $M_{\mathrm{mol}} / L_{\mathrm{B}}$ ratios, but the atomic gas content is similar for the two samples. The analysis of the luminosity-size and mass-luminosity relations shows similar trends for both families, the main difference being the almost total absence of big, bright and massive galaxies among the family of isolated systems, together with the almost total absence of small, faint and low mass galaxies among the perturbed systems. All these aspects indicate that the evolution induced by interactions with neighbors would proceed from late, small, faint and low mass Spirals to earlier, bigger, more luminous and more massive spiral and lenticular galaxies, producing at the same time a larger fraction of barred galaxies but preserving the same relations between global parameters. The properties we found for our sample of isolated galaxies appear similar to those of high redshift galaxies, suggesting that the present-day isolated galaxies could be quietly evolved, unused building blocks surviving in low density environments.
\end{abstract}

Key words. galaxies: interactions - galaxies: evolution - galaxies: spiral

\section{Introduction}

Galaxies are most commonly found in aggregates of different densities. Inside these aggregates, the observed morphology and the segregation of types (Dressler et al. 1997; Fasano et al. 2000) may have been determined by the local density of matter and by the subsequent interactions between galaxies. Exchanges and interaction with its neighbors can affect the galaxies global properties and their star formation rates, as

\footnotetext{
Send offprint requests to: M. Moles, e-mail: moles@iaa.es

* Tables 1 and 2 are only available in electronic form at http://www. edpsciences.org
}

shown by the members of dense groups or pairs (Moles et al. 1994a), even if the effects, in general, are not as dramatic as they are in the strongest cases (Moles et al. 1994b; Márquez \& Moles 1999).

Another way to look into the effects of interaction on the overall equilibrium of a galaxy is through the analysis of the relations found between the structural parameters for different families of galaxies. The E/S0 cluster (Faber et al. 1987; Davis \& Djorgovski 1987; Jørgensen et al. 1996), and field galaxies (Treu et al. 2001) satisfy the Fundamental Plane (FP) relation, even if its form is not the same for both families. The Spiral (S) galaxies satisfy the Tully-Fisher (TF) relation. It has 
been indicated that the TF relation defined by isolated spiral galaxies presents a smaller scatter than non-isolated galaxies (Márquez \& Moles 1996; Márquez et al. 2002; van Kannappan et al. 2002).

To find isolated galaxies, a physically grounded and operational definition of isolation has to be given. Often the term field galaxies refers to systems that are not in dense aggregates, but frequently they are still under the influence of neighboring galaxies and cannot be considered as genuinely isolated objects (see for example Márquez et al. 2002). The first systematic compilation of isolated galaxies was made by Karachentseva (1973), who used as the main criterion that they do not show companions within 20 diameters in the PSS plates. Later, Turner \& Gott (1976), while searching for groups of galaxies, found that some of the galaxies could not be assigned to any of the identified groups. It was argued that they would trace a cosmological homogeneously distributed population of galaxies. This conclusion was however challenged by Huchra $\&$ Thuan (1977) who used a deeper sample $(m \leq 15.7$ instead of $m \leq 14$ ). Vettolani et al. (1986) adopted stricter criteria to find isolated galaxies. Their conclusion was that it was very unlikely that there was a cosmological component of galaxies without any kind of clustering (at least at small redshift). But an important observation was the detection of galaxies in such poor environments that they had very probably evolved without any external perturbation, as if they were isolated. In that perspective what is needed is an operational definition of isolation that could lead to identifying galaxies that have evolved free from external influences for most of their lives, a point of view adopted by Márquez \& Moles (1996) and Márquez et al. (1999). In this context, it was noticed that non-axisymmetric structures like bars, tails or plumes, which are usually explained invoking gravitational interaction with companions or satellites, can also be present in isolated galaxies, posing the problem of their origin in the absence of sizable interactions (Moles et al. 1994, 1995).

In the present work we discuss the criteria used to find isolated galaxies and build a statistically well-defined sample to analyze their properties. Due to the lack of redshift information for most of the faint, possible perturbers, the final sample is by no means complete, our main goal being to keep only truly isolated galaxies, discarding all the suspect systems. In the last section we compare the properties of the family of the isolated galaxies with those of confirmed perturbed galaxies.

We also consider the similarity of some of the properties of present-day isolated systems with those reported for the presumed building blocks in the distant Universe (Ferguson et al. 2003; Trujillo et al. 2003). Stellar systems situated in regions of very low density would have evolved differently from galaxies in aggregates without a significant contribution from the surroundings and, therefore, they might be similar to those original building blocks.

\section{Isolation criteria}

From an operational point of view, we will consider a galaxy to be isolated when its evolution in terms of structure and stellar content has been dominated by internal forces for most of its life. Or, in other terms, when the external forces are judged to be unable to have produced noticeable changes in at least the last $2 \times 10^{9}$ years. As found by Athanassoula (1984) and Byrd $\&$ Howard (1992), an external interaction can only have influence on the structure of a given system when the corresponding tidal force amounts to $\geq 1 \%$ of the internal force.

The results of numerical simulations of encounters between galaxies can be described in terms of a tidal perturbation parameter (see Byrd \& Howard 1992). This gives the ratio between the tidal force exerted by the perturber, $\mathrm{P}$, on the primary galaxy, $\mathrm{G}$, and the internal force per unit mass in the outer parts of the primary. Given a galaxy of mass $M_{\mathrm{G}}$ and size $R$, and a perturber of mass $M_{\mathrm{P}}$ with a closest approach pericenter distance given by $b$, that ratio is

$\frac{F_{\text {ext }}}{F_{\text {int }}} \propto\left(\frac{M_{\mathrm{P}}}{M_{\mathrm{G}}}\right) \times\left(\frac{R}{b}\right)^{3}$.

In this expression the masses of the galaxies can be evaluated using the diameters or the luminosities. Actually, since there is a relation between luminosity and size, both parameters should lead to similar results. We preferred to use the magnitudes here instead of the sizes (used for instance by Dahari 1984) since they are known for a larger number of galaxies and are easier to correct. Indeed, it is assumed that $M / L$ is reasonably similar for all the galaxies. Admittedly this is a rough hypothesis but appropriate since the final criteria for isolation we are going to adopt is likely insensitive to the differences in $M / L$ from galaxy to galaxy.

Regarding the pericenter parameter, $b$, Icke (1985), when studying the influence on the gas in the disk of a galaxy of the flyby of another galaxy, found that the maximum pericenter distance still able to trigger star formation in the gas disk is given by

$b=\left(4 \pi g \frac{v}{s} \mu\right)^{1 / 3} r$

where $g$ is a geometrical factor describing the encounter that ranges between 0 and $1, v$ the gas speed at the distance $r$ of the center of the disk, $s$ the sound speed at the same distance, and $\mu$ the ratio of the perturber's mass to the central galaxy mass. The factor in brackets is, at most, of the order of a few, and therefore $b$ is, at most, of the order of or a few times the radius of the perturbed galaxy. Thus, only close encounters could produce important effects in the internal dynamics of the gas in the other system, in agreement with the results by Athanassoula (1984) and Byrd \& Howard (1992).

Since the pericenter parameter $b$ cannot be directly estimated, we adopted instead the projected distance $D_{\mathrm{p}}$ between the galaxy and the perturber on the plane of the sky at the primary's distance. Thus, the final expression for the perturbation parameter is

$f=\log \left(\frac{F_{\text {ext }}}{F_{\text {int }}}\right)=3 \log \left(\frac{R}{D_{\mathrm{p}}}\right)+0.4 \times\left(m_{\mathrm{G}}-m_{\mathrm{P}}\right)$

where $m_{\mathrm{G}}$ and $m_{\mathrm{P}}$ are the apparent magnitudes of the primary and perturber galaxies, respectively.

In fact $D_{\mathrm{p}}$ can be greater than the impact parameter, $b$, depending on the orbit type, the orientation of the line of nodes 


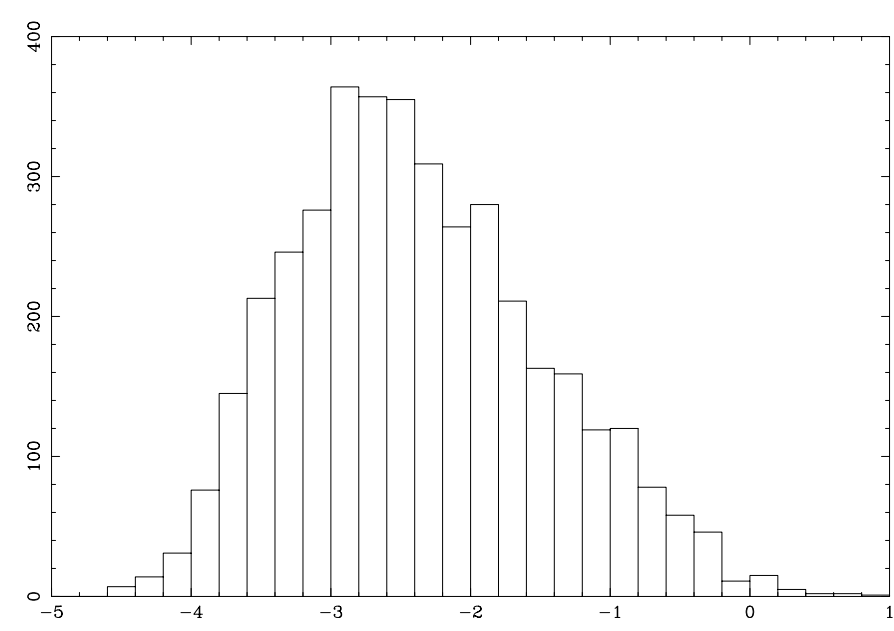

Fig. 1. The distribution of the $f$-values for galaxies in the Coma Cluster.

with respect to the observer and the position of the perturber on its orbit around the primary, all quantities totally unknown. In those cases a galaxy would be judged more isolated (i.e., smaller $f$-values) than it really is, which would compromise our selection criterion. The error in $f$ assuming $D_{\mathrm{p}}$ instead of $b$ is $3 \times \log \left(D_{\mathrm{p}} / b\right)$. As we will see, given that we are going to make a statistical use of $f$, and the restrictive limits we impose in order to consider a galaxy as isolated, the adopted criterion is robust enough and only in very extreme (i.e. very improbable) cases, a perturbed system might be considered as isolated.

The theoretical results by Athanassoula (1984), Byrd \& Howard (1992) and Icke (1985) consistently show that values of $f \geq-2$ are required to produce sizable effects on a given disk galaxy. However, before fixing the limit $f$-value to consider a galaxy isolated, we must consider the fact that we are using rather rough estimates for the masses of the galaxies and for the pericenter distance. Thus, systems with $f$-values now observed to be smaller than -2 could had have presented larger values in the past, depending on the details of the orbit and of the reaction to the interaction.

To evaluate the typical $f$ values existing in well defined galaxy aggregates, we studied their distribution for the galaxies of the Coma Cluster. As far as it can be considered to be in a stationary state, the range of $f$-values we find at a given moment should be statistically representative of the values it can have over time, and it would be possible to extract conclusions that are valid for the whole duration of the stationary state. Therefore we took data from Godwin et al. (1983), and considered all the galaxies in Coma with photometry in the $B$ and $R$ bands, a total of 4075 objects. They cover a wide range of magnitude and size. The distribution of the $f$-parameter values is presented in Fig. 1. The median value of the distribution is -2.7 and, as it can be seen in the figure, there are no galaxies with $f<-4.5$.

In view of these results for Coma, it seems safe to adopt the formal criterion $f \leq-4.5$ to select what we will call isolated galaxies.

From the preceding considerations it is clear that the $f$ cutoff value is probably too strict for situations where a candidate galaxy has only a few possible companions at most. Thus it is robust enough to cope with probable errors in the different observable parameters entering the definition and ensure that all the selected objects are actually isolated. From expression (3), it is simple to verify that errors of $20 \%$ in size and/or luminosity translate into errors of only a few tenths in $f$ at most, still very far from critical values. Similarly, even a ratio between the projected distance and the pericenter distance as large as $D_{\mathrm{P}} / b=3$ would produce an $f$-value greater than the true value by 1.4 units, still far from critical values. Only for very eccentric orbits, and when the perturber is around the apocenter, our criterion would fail and could select false isolated galaxies.

Another aspect to consider is the possibility of inducing biases in the family of the selected objects, given the dependency of $f$ on the luminosity and size. A galaxy might have a different probability of being taken as isolated for a fixed population of possible companions. However, given a direct relation between size and luminosity, we expect only a small net effect on $f$. Thus, considering $L \propto R^{2}$, the difference in the $f$-values produced by a given perturber on galaxies with luminosities of the ratio $1 / 1.5$ would amount to only 0.17 , well inside the uncertainties in $M / L$ or any other parameter, and unable to approach critical values.

Given the preceding considerations we do not expect strong biases in the family of selected isolated galaxies.

\subsection{Comparison with previous criteria}

Karachentseva (1973) considered a galaxy of diameter $d_{\mathrm{i}}$ as isolated when there were no companions with size, $d_{c}$, between $1 / 4$ and $4 \times d_{\mathrm{i}}$, within $20 \times d_{\mathrm{c}}$. To compare this with our criteria we have to assume that the luminosity is proportional to some power of the size, say $L \propto R^{2}$. It is easy to verify that both criteria are similar. However, Karachentseva's criterion does not exclude the possibility of small, faint perturbers that could have some effect if they are close enough to the primary galaxy.

Márquez \& Moles (1996) defined a galaxy as isolated if there are no companions within a projected radius of $0.5 \mathrm{Mpc}$, and with a relative redshift less than $500 \mathrm{~km} \mathrm{~s}^{-1}$. They also made a visual inspection of the POSS images to search for faint companions. Applying the $f$-value criterion to their data, we find that over $90 \%$ of the galaxies considered isolated by Márquez \& Moles (1996) are also isolated using our criterion.

Vettolani et al. (1986) considered as isolated the galaxies without companions with $m \leq 14.5$, within a radius that varies with the distance to the galaxy to keep constant the probability of finding a galaxy in a given volume. The work was complemented with a search for fainter companions in the POSS plates, without a clearly stated objective criterion. We found that 15 out of the 43 galaxies in their sample have faint companions. We applied our $f$-parameter selection method to the other 28 galaxies, to find that only 2 of them would be considered as isolated with our criterion. This is a clear illustration of the problems that can be encountered when the difference between field and isolated galaxies is not clearly stated. 


\section{The selection process}

The starting point of our search was the CfA catalog of galaxies (Huchra et al. 2000). Our aim was to build a volumelimited, statistically meaningful sample of isolated spiral galaxies. To that end we first selected all the galaxies classified as disk galaxies (Spiral and Lenticular), with $c z \leq 5000 \mathrm{~km} \mathrm{~s}^{-1}$. Only objects at high galactic latitudes, $|b| \geq 40^{\circ}$, were retained, to avoid problems with the extinction correction. Finally we considered only objects with declinations north of $-2.5^{\circ}$. 1706 galaxies satisfy all these conditions. The properties of these galaxies were extracted from the LEDA database (Paturel et al. 1997). The distances were calculated using the LEDA kinematical distance modulus, corrected for extinction and Virgocentric flow.

Each galaxy was then searched for companions in the same LEDA catalog, complete to $m=18$. The identification of the CfA objects with LEDA objects is not always straightforward since offsets in the coordinates up to $1^{\prime}$ are not infrequent. When there was a possibility of misidentification we decided after visual inspection. Once all the CfA objects were identified in LEDA, we started the search for companions. The searching field was limited to that defined by the maximum distance at which a bright (massive) galaxy with $M=-23$ would produce a value of $f=-4.5$. Special care was taken to avoid confusion with extended objects that are not galaxies, and with double or distorted star images that were taken as galaxies in the preliminary version of the catalog we used.

We also imposed two more restrictions, on size and luminosity, to eliminate as many background objects as possible. The suspected companions were retained only if, at the distance of the parent galaxy, they would be larger than $2 \mathrm{kpc}$ in diameter, and brighter than $M_{\mathrm{P}}=-12$. For some faint galaxies present in the search field no magnitudes were listed in the LEDA catalog. In these cases we calculated the apparent magnitude that those objects would have to produce $f=-4.5$, and we compared it with the estimation from visual inspection. Only companions found brighter than that value were retained. Using those restrictions we could reject most of the possible companions. For the doubtful cases the central galaxy was taken as perturbed. In a final step we discarded all the companions for which the (known) redshift is different from that of the primary galaxy by more than $500 \mathrm{~km} \mathrm{~s}^{-1}$. The $f$ values for all the possible perturbers within the search volume were computed, and only those with $f>-4.5$ and satisfying the conditions on size and luminosity were finally taken as possible perturbers. The above selection process reduced the list of candidate galaxies to 329 .

We then examined the DSS data looking for the presence of possible perturbers with $m_{\mathrm{P}}<18$. The process was only applied to the volume where those faint galaxies would still produce $f \geq-4.5$. The size and magnitude of all the possible perturbers were measured, and the size and luminosity criteria applied. More than 100 galaxies were found to have companions. At the end of the process a list of 203 isolated $\mathrm{S}$ and S0 galaxies was produced. This represents less than $12 \%$ of the initial sample. We insist that the list is not complete since all the galaxies with faint companions $\left(m_{\mathrm{P}}<18\right.$ and $\left.f \geq-4.5\right)$ without known redshift were discarded. Besides that catalogue of isolated galaxies we selected another sample of non-isolated galaxies containing systems having companions with $f>-2$, and $\Delta z<500 \mathrm{~km} \mathrm{~s}^{-1}$. A total of 130 objects were extracted from the original sample. This sample will be used as a comparison for the properties of isolated galaxies. In the text, we shall refer to this sample as perturbed galaxies.

\section{Properties of disk isolated and perturbed galaxies}

The database we used for the properties of the galaxies is the LEDA Catalogue. The identification and main properties of the isolated and perturbed galaxies are presented in Tables 1 and $2^{1}$ respectively.

The data presented in these tables have been extracted from various sources, as described in the following. To standardize the information contained in our catalogue we extracted from the LEDA (Paturel et al. 1997) catalogue for each galaxy: PGC number, morphological type code $t$, the geometrical parameters at the $25 \mathrm{mag} \operatorname{arcsec}^{2}$ isophote $\log D_{25}$ and $\log r_{25}$, the corrected colors $(U-B)_{0}$ and $(B-V)_{0}$. the mean surface brightness at the same isophote $\mu_{25}$, the kinematical parameters $W_{20}$ (the $21-\mathrm{cm}$ line width at $20 \%$ of the peak), $\log \sigma$ (velocity dispersion), $\log v_{\mathrm{m}}$ (the maximun rotational velocity), the redshift $c z$, the kinematic distance modulus $(m-M)_{\text {cin }}$, the blue corrected absolute magnitude $M_{\mathrm{B}}$, the far infrared magnitude $m_{\mathrm{FIR}}$ and the $21-\mathrm{cm}$ line magnitude $m_{21}$. The distance moduli are mainly derived from redshifts, corrected for Virgocentric inflow and adopting $H_{0}=70 \mathrm{~km} \mathrm{~s}^{-1} \mathrm{Mpc}^{-1}$. When redshift was not available, we used the photometric distance modulus, if present in LEDA. The absolute values were calculated using these distance moduli. The entry coded as FIR-B in Table 1 corresponds to $M_{\mathrm{FIR}}-M_{\mathrm{B}}$.

Information lacking in LEDA for some galaxies was completed using the ADS bibliographic archive, the NED database or the SIMBAD service of the Strasbourg Centre of Données Stellaires (CDS), as indicated in the references of Table 1. They are:

1) the HI masses in solar units, calculated from $m_{21}$, with the expression:

$\log M_{\mathrm{HI}}=5.37-0.4\left(m_{21 \mathrm{c}}-17.4\right)+2 \log d$

or calculated from 21-cm fluxes $S_{21}$, in $\mathrm{Jy} \mathrm{km} \mathrm{s}^{-1}$, by the formula:

$\log M_{\mathrm{HI}}=5.37+\log S_{21}+2 \log d ;$

2) the molecular gas masses in solar units from $\mathrm{CO}(1-0)$ line fluxes $\left(S_{\mathrm{CO}}\right.$ in $\left.\mathrm{Jy} \mathrm{km} \mathrm{s}^{-1}\right)$ by the formula:

$\log M_{\mathrm{mol}}=4.17+2 \log d+\log S_{\mathrm{CO}}$

that implicitly assumes a constant $\mathrm{CO} / \mathrm{H}_{2}$ conversion factor $\chi=N\left(\mathrm{H}_{2}\right) / I_{\mathrm{CO}}=2.3 \times 10^{20} \mathrm{~mol} / \mathrm{K} \mathrm{km} \mathrm{s}^{-1}$ (Strong 1988);

$M_{\text {mol }}$ includes the helium mass fraction, equal to $36 \%$ of the $\mathrm{H}_{2}$ mass.

1 Only available in electronic form at http://www . edpsciences.org. 
Table 3. Comparison of the main properties of Isolated (Is) and Perturbed (Pt) galaxies. The statistical parameter $z$ from the U-test is given in the third column. In the last three columns we give the median values for the three morphological bins defined in the text. The first row contains the results for the morphological types, the numbers in parentheses corresponding to the number of galaxies used in the comparison. The second row gives the fraction of galaxies with bars. The other rows correspond to the other properties we considered.

\begin{tabular}{|c|c|c|c|c|c|}
\hline & Status & $z$-value & S0 & Early Sp & Late $\mathrm{Sp}$ \\
\hline \multirow[t]{2}{*}{ Type } & Is (196) & 2.79 & $21.4 \%$ & $36.7 \%$ & $41.8 \%$ \\
\hline & Pt (129) & & $31.0 \%$ & $43.4 \%$ & $25.6 \%$ \\
\hline \multirow[t]{2}{*}{ Bars } & Is (64) & - & $15.7 \%$ & $34.9 \%$ & $38.2 \%$ \\
\hline & $\mathrm{Pt}(66)$ & & $39.2 \%$ & $71.1 \%$ & $41.2 \%$ \\
\hline \multirow[t]{2}{*}{$M_{\mathrm{B}}$} & Is (203) & 4.74 & $-17.88(42)$ & $-19.55(72)$ & $-19.27(89)$ \\
\hline & Pt (130) & & $-19.60(40)$ & $-19.90(56)$ & $-19.73(34)$ \\
\hline \multirow[t]{2}{*}{$\log D_{25}$} & Is (203) & 6.05 & $0.90(42)$ & $1.21(72)$ & $1.20(89)$ \\
\hline & Pt (130) & & $1.28(40)$ & $1.38(56)$ & $1.35(34)$ \\
\hline \multirow[t]{2}{*}{$(U-B)$} & Is (45) & 3.84 & $0.22(11)$ & $0.03(19)$ & $-0.12(15)$ \\
\hline & $\mathrm{Pt}(67)$ & & $0.40(26)$ & $0.15(27)$ & $-0.07(14)$ \\
\hline \multirow[t]{2}{*}{$(B-V)$} & Is (58) & 3.69 & $0.76(11)$ & $0.66(19)$ & $0.51(28)$ \\
\hline & $\mathrm{Pt}(78)$ & & 0.85 (29) & $0.68(31)$ & $0.53(18)$ \\
\hline \multirow[t]{2}{*}{$\mu_{25}$} & Is (203) & 2.73 & $23.29(42)$ & $23.04(72)$ & $23.39(89)$ \\
\hline & $\mathrm{Pt}(130)$ & & $23.50(40)$ & $23.38(56)$ & $23.38(34)$ \\
\hline \multirow[t]{2}{*}{$\log v_{\mathrm{m}}$} & Is (149) & 3.99 & $1.87(13)$ & $2.20(55)$ & $2.09(81)$ \\
\hline & $\mathrm{Pt}(94)$ & & $2.21(15)$ & $2.31(48)$ & $2.13(31)$ \\
\hline \multirow[t]{2}{*}{$\log \sigma$} & Is (23) & 3.15 & $2.06(10)$ & $2.23(6)$ & $1.99(7)$ \\
\hline & $\mathrm{Pt}(46)$ & & $2.25(26)$ & $2.18(16)$ & $2.05(4)$ \\
\hline \multirow[t]{2}{*}{$W_{20}$} & Is (139) & 5.09 & $164(13)$ & $290(53)$ & $240(73)$ \\
\hline & $\mathrm{Pt}(87)$ & & $327(14)$ & $386(45)$ & $264(28)$ \\
\hline \multirow[t]{2}{*}{$M_{\mathrm{FIR}}-M_{\mathrm{B}}$} & Is (104) & 3.02 & $-0.80(11)$ & $-0.80(45)$ & $-0.31(48)$ \\
\hline & $\mathrm{Pt}(44)$ & & $-1.06(1)$ & $-0.34(27)$ & $-0.25(16)$ \\
\hline \multirow[t]{2}{*}{$\log \left(M_{\mathrm{HI}} / L_{\mathrm{B}}\right)$} & Is (151) & 0.91 & $-0.74(14)$ & $-0.67(55)$ & $-0.46(82)$ \\
\hline & Pt (93) & & $-1.17(15)$ & $-0.66(47)$ & $-0.38(31)$ \\
\hline \multirow[t]{2}{*}{$\log \left(M_{\mathrm{mol}} / L_{\mathrm{B}}\right)$} & Is (21) & 2.61 & $-0.89(2)$ & $-0.64(5)$ & $-1.15(14)$ \\
\hline & $\mathrm{Pt}(20)$ & & - & $-0.62(13)$ & $-0.62(7)$ \\
\hline
\end{tabular}

When only mass values were available in the references, they were scaled to the distances assumed here. When data for a single galaxy were available in several catalogues, we compared the mass values producing a weighted mean value. When both upper limits and detections were available, only detections were used to compute mean values. Moreover, when several upper limits were available, only the lowest value was adopted. All the above mass data were normalized by the total blue luminosity in solar units, calculated from $M_{\mathrm{B}}$.

\subsection{Main properties of the samples}

The first aspect we considered was the distribution of the galaxies in both samples by morphological types (Fig. 2). The differences between the isolated and perturbed galaxy distributions are apparent. Given the clear non-normality of the distributions, we applied the Mann-Whitney U-test. The results indicate that the two distributions are different with a significance level $>99.5 \%$. The largest differences are found for Sc galaxies, that are more abundant among isolated galaxies (in agreement with earlier results by Márquez 1994; Márquez \& Moles 1996; Márquez et al. 1999), and for S0 galaxies, that are more abundant among perturbed galaxies (see also Table 3 ).

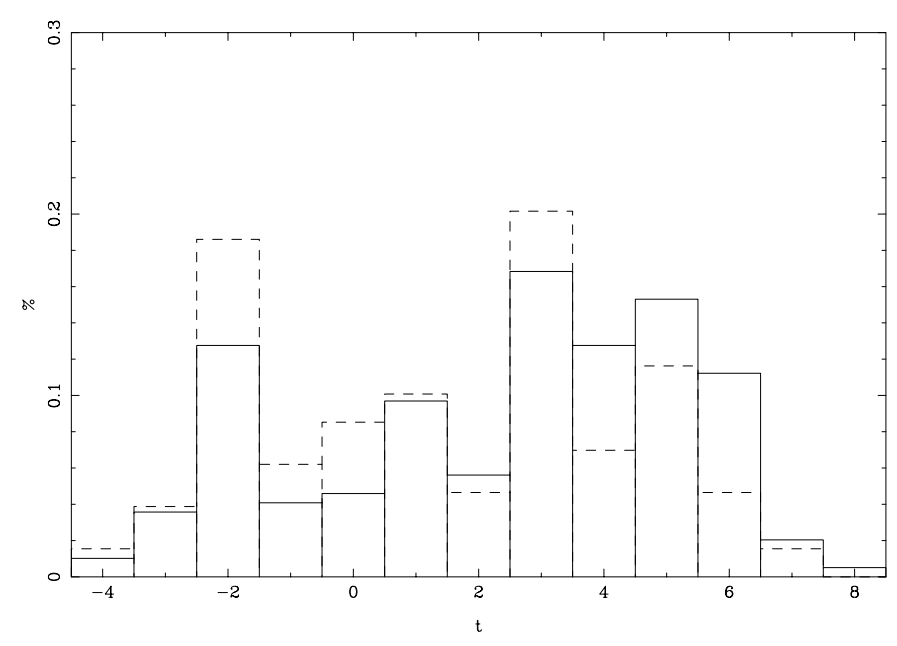

Fig. 2. The normalized distributions of morphological types for Isolated (solid line) and Perturbed (dashed line) galaxies.

To compare all the other properties of isolated and perturbed galaxies we grouped them into three morphological categories, S0 $(-4.5<t \leq-0.5)$, early Sp $(-0.5<t \leq 3.5)$ and late $\mathrm{Sp}(t>3.5)$. 
It appears that barred galaxies are far more abundant (about twice) among perturbed galaxies. The difference is very marked for Lenticular and early Sp types, whereas there is no difference for late Sp galaxies (see Table 3). We also compared all the other catalogued properties of the two samples. The differences were tested with the Mann-Whitney U-test. We found that the two samples are different at a highly significant level $(>99.5 \%)$ in all the main catalogued properties, including the absolute blue magnitude, $M_{\mathrm{B}}$, the infrared luminosity normalized to the B luminosity, $M_{\mathrm{FIR}}-M_{\mathrm{B}}$, the size, $D_{25}$, the color indexes $(U-B)$ and $(B-V)$, the mean surface brightness within the isophote $25, \mu_{25}$, the maximum velocity rotation of the gas, $v_{\mathrm{m}}$, the stellar central velocity dispersion, $\sigma$, and the $21-\mathrm{cm}$ line width at $20 \%$ of the peak, $W_{20}$. In Table 3 we give the median values of those properties for the different families.

The only property that appears to be similar for both samples is the amount of atomic gas as measured by $\log \left(M_{\mathrm{HI}} / L_{\mathrm{B}}\right)$. Looking at the different morphological bin there is a hint of a possible difference for lenticular galaxies.

It can be seen that the isolated galaxies are smaller, less luminous and bluer than the perturbed systems. We also notice the consistency of the results regarding the dynamical variables. $V_{\max }, \sigma$ and $W_{20}$ are smaller for the isolated galaxies. They also present lower FIR luminosity and molecular gas content. Notice that the same trend is seen in all the morphological bins we considered, even if the largest and more significant differences are found in most cases for the lenticular and early Sp galaxies.

Since the content of molecular gas is not given in LEDA we searched the literature using the same references and approach as Bettoni et al. (2003, the references are given in the Notes of Tables 1 and 2). We found data for only 21 isolated and 20 perturbed galaxies. We found that the perturbed galaxies have higher $M_{\mathrm{mol}} / L_{\mathrm{B}}$ values, the difference being statistically significant at $>99.5 \%$. Notice that the difference arise only in the late SP morphological bin.

We analyzed the relation between global structural parameters. We found that both families satisfy the same LuminositySize relation (Fig. 3). We notice the almost absence of bright and large isolated galaxies, together with the almost absence of small and faint perturbed galaxies. This tendency cannot be due to the selection criteria, based on $f$ values, and depending on sizes and luminosity as in Eq. (3). More massive and less extended systems are less subject to perturbations by the surrounding galaxies. One may expect that galaxies selected on the basis of a lower $f$ could be biased toward smaller but brighter systems. On the contrary, isolated galaxies in our sample appear smaller, i.e. producing smaller $f$-values, but fainter, i.e. producing higher $f$-values.

In Fig. 4 we present the $M$ versus $L$ relation. The masses have been estimated using a central, point-like mass model, with $M\left(M_{\odot}\right)=2.3265 \times v^{2} R_{25}\left(v\right.$, from LEDA, in $\mathrm{km} \mathrm{s}^{-1}$ and $R_{25}$ in $\mathrm{kpc}$ ). It can be seen in the figure that both families, isolated and perturbed galaxies, define very approximately the same relation, and share an important region in the diagram. The differences arise because there are no isolated galaxies with high mass (and luminosity), whereas there are no perturbed galaxies with low mass (and luminosity).

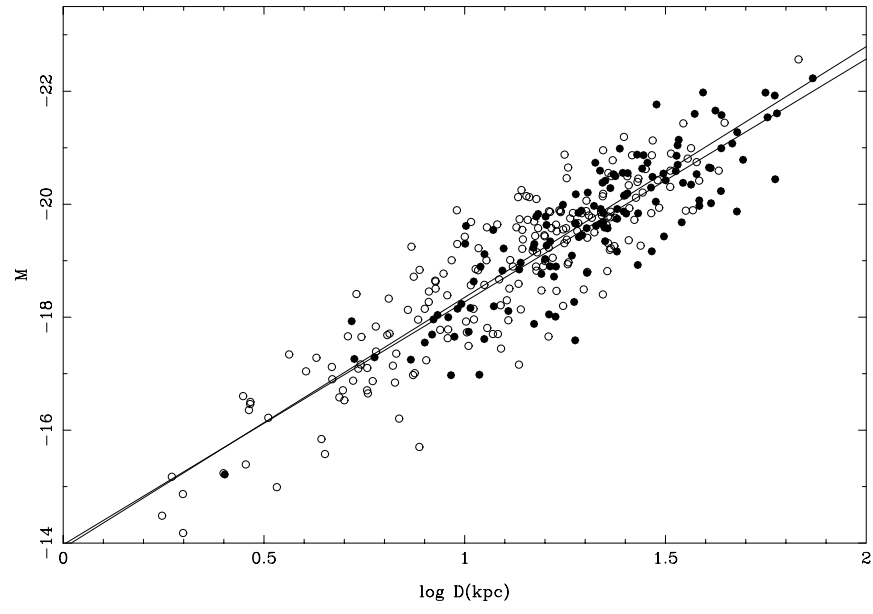

Fig. 3. The luminosity-size relation for Isolated (empty circles) and Perturbed (filled circles) galaxies. The lines are the best fit to the two families.

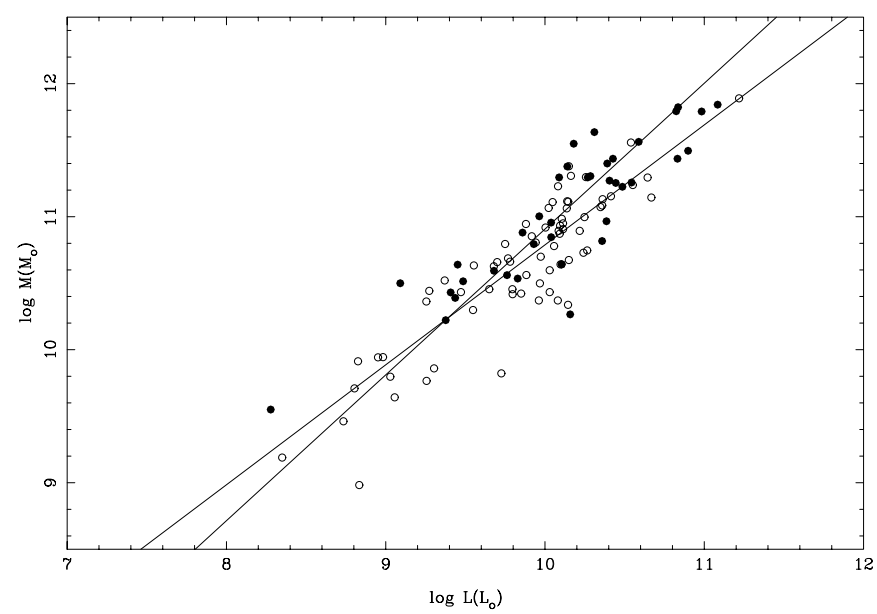

Fig. 4. The mass-luminosity relation for Isolated and Perturbed galaxies. Same symbols as in Fig. 3. The rotation velocity was corrected for inclination. Only galaxies not later than Scd, with inclinations between 40 and 70 degrees have been plotted. The lines are the fits to both families.

The Tully-Fisher relation for $\mathrm{S}$ galaxies in both samples is presented in Fig. 5. Even if both families follow the standard TF relation (Tully \& Pierce 2000), the scatter is important and no conclusion about possible differences can be extracted before more homogeneous and accurate data are available. Apparently the presence of bars does not have any influence on the position of a galaxy in the T-F diagram, in agreement with the results reported by Courteau et al. (2003).

\section{Conclusions}

We used a well-defined, physically meaningful criterion to define isolated galaxies. We performed a search for isolated galaxies using as the parameter the logarithmic ratio $f$ between the internal and tidal forces at the outskirts of a given galaxy. The adopted limit, $f<-4.5$, was checked with results from numerical simulations (Byrd \& Howard 1992), and with data from the Coma Cluster. Similarly, a sample of perturbed 


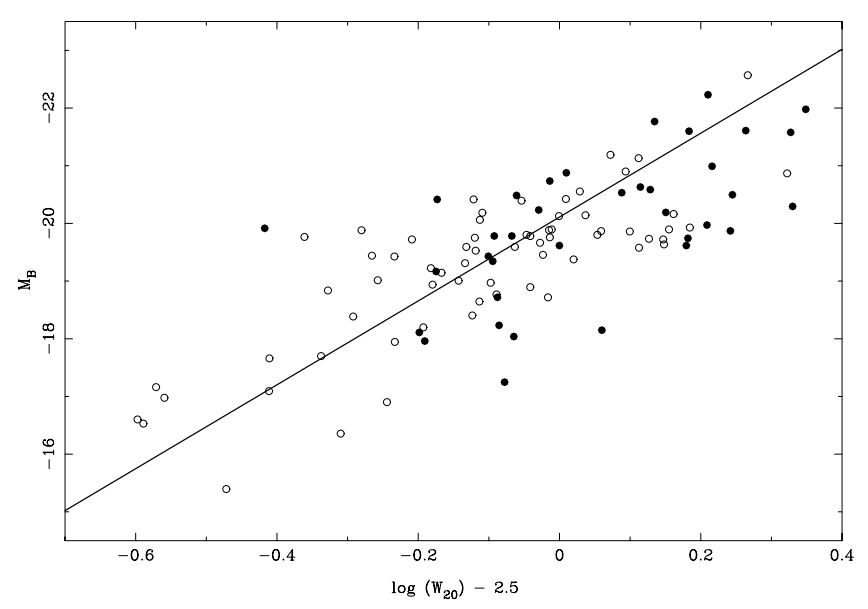

Fig. 5. The Tully-Fisher relation for Isolated and Perturbed galaxies. Same symbols and galaxies as in Fig. 4. The line represents the fit to the T-F relation as given by Tully \& Pierce (2002).

galaxies was defined comprising galaxies with confirmed companions satisfying $f \geq-2$.

Comparison of the properties of the galaxies in both samples was made using the Mann-Whitney U-test. The first result to notice is the significant differences in morphological types, with Sc types being more abundant among isolated galaxies, whereas S0 galaxies are significantly more abundant among perturbed systems. We also found that barred galaxies are more frequent (about twice) among perturbed than among isolated galaxies except for late type Spiral galaxies.

They also differ in all the properties we have examined, except in atomic gas content, with similar values of the ratio $M_{\mathrm{HI}} / L_{\mathrm{B}}$. The isolated galaxies appear to be smaller and fainter, with bluer color indices. They also have less molecular gas and smaller FIR luminosity per unit of blue luminosity. The dynamical parameters are also consistently smaller for isolated galaxies. Even if the differences are appreciable for all the families (S0, early Sp and late Sp), the biggest differences are for S0 and early Sp. The exception is in molecular gas content, for which the maximum difference is found for late-type Sp galaxies.

The $M-L$ and $M$-size relations consistently show the absence of large, luminous and massive systems among the isolated galaxies. Moreover, we also found the almost complete absence of perturbed systems with small sizes, low luminosities and low masses. We also found that barred galaxies do not occupy a particular region of the T-F diagram.

In view of these results it is tempting to consider the differences as arising from the different evolutionary conditions. Our results indicate that the gravitational interaction in aggregates would produce evolution from late Spiral, relatively faint and low mass galaxies, toward earlier more luminous and massive Spiral and Lenticular types. This would also favor the formation of bars in early-type Spirals and Lenticulars. However, the relations between global parameters are similar for both families, even if they tend to occupy different regions in the corresponding plots (see Figs. 2-4)

Since the isolated galaxies would not have had the opportunity to accrete other systems and grow in the way the hierarchical models predict, they would still be similar today to the high redshift systems supposed to be the building blocks for the formation of larger galaxies. This view is consistent with the results reported by Ferguson et al. (2003) showing that the sizes of galaxies at $z \approx 4$ are smaller than nearby luminous galaxies. If, as it is generally assumed, cannibalism and accretion in the early phases of galaxy evolution are important to fix the final global properties such as size, luminosity and mass, then present day isolated galaxies would be the left-over fragments in that early process of galaxy formation.

Acknowledgements. J.V., M.M., I.M. and J.M. acknowledge financial support from the Spanish Ministerio de Ciencia y Tecnología through grants PB98-0684, AYA2002-01241 and AYA2001-2089 and from the Junta de Andalucía, grant TIC-144. GG has made use of funds from the University of Padova (Fondi 60\% - 2002). We thank Dr. G. Paturel for allowing us to use the new LEDA catalogue prior to publication.

\section{References}

Athanassoula, E. 1984, Phys. Rep., 114, 321

Bettoni, D., Galletta, G., \& García-Burillo, S. 2003, A\&A, 405, 5

Byrd, G. G., \& Howard, S. 1992, AJ, 103, 1089

Courteau, S., Andersen, D. R., Bershady, M. A., MacArthur, L. A., \& Rix, H.-W. 2003, ApJ, 594, 208

Djorgovski, S., \& Davis, M. 1987, ApJ, 313, 59

Dressler, A., Oemler, A. Jr., Couch, W. J., et al. 1997, ApJ, 490, 577

Dahari, O. 1984, AJ, 89, 966

Faber, S. M., Dressler, A., Davies, R. L., Burstein, D., \& Lynden-Bell, D. 1987, Nearly Normal Galaxies, From the Planck Time to the Present, 175

Fasano, G., Poggianti, B. M., Couch, W. J., et al. 2000, ApJ, 542, 673

Ferguson, H. C., Dickenson, M., Giavalisco, M., et al. 2003 [arXiv: astro-ph/0309058]

Godwin, J. G., Metcalfe, N., \& Peach, J. V. 1983, MNRAS, 202, 113

Huchra, J., et al. 2000, CfA Redshift Catalog

Huchra, J., \& Thuan, T. X. 1977, ApJ, 216, 694

Icke, V. 1985, A\&A, 144, 115

Jørgensen, I., Franx, M., \& Kjærgaard, P. 1996, MNRAS, 280, 167

van Kannappan, S. J., Fabricant, D. G., \& Franx, M. 2002, AJ, 123, 2358

Karachentseva, V. E. 1973, Astrofizicheskie Issledovaniia Izvestiya Spetsial'noj Astrofizicheskoj Observatorii, 8, 3

Márquez, I. 1994, Ph.D., Universidad de Granada

Márquez, I., \& Moles, M. 1996, A\&AS, 120, 1

Márquez, I., \& Moles, M. 1999, A\&A, 344, 421

Márquez, I., Durret, F., González-Delgado, R. M., et al. 1999, A\&AS, 140,1

Márquez, I., Masegosa, J., Moles, M., et al. 2002, A\&A, 393, 389

Moles, M., Márquez, I., Masegosa, J., et al. 1994, ApJ, 432, 135

Moles, M., del Olmo, A., Perea, J., et al. 1994, A\&A, 285, 404

Moles, M., Marquez, I., \& Pérez, E. 1995, ApJ, 438, 604

Paturel, G., Andernach, H., Bottinelli, L., et al. 1997, A\&AS, 124, 109

Strong, A. W., Bloemen, J. B. G. M., Dame, T. M., et al. 1988, A\&A, 207,1

Treu, T., Stiavelli, M., Bertin, G., Casertano, S., \& Møller, P. 2001, MNRAS, 326, 237

Trujillo, I., Rudnick, G., Rix, H.-W., et al. 2003 [arXiv: astro-ph/0307015]

Tully, R. B., \& Pierce, M. J. 2000, ApJ, 533, 744

Turner, E. L., \& Gott, J. R. 1976, ApJS, 32, 409

Vettolani, G., de Souza, R., \& Chincarini, G. 1986, A\&A, 154, 343 


\section{Online Material}


Table 1. Main properties of the Isolated galaxies. All the propertis were extracted from LEDA except the molecular gas content.

\begin{tabular}{|c|c|c|c|c|c|c|c|c|c|c|c|c|c|c|c|c|c|}
\hline PGC & $t$ & $\begin{array}{r}\log D_{25} \\
\quad\left(0{ }^{\prime} 1\right)\end{array}$ & $\begin{array}{r}\log r_{25} \\
(0.1)\end{array}$ & $(U-B)_{0}$ & $(B-V)_{0}$ & $\begin{array}{c}\mu_{25} \\
\left(\mathrm{mag} / \operatorname{arcsec}^{2}\right)\end{array}$ & $\begin{array}{r}W_{20} \\
\left(\mathrm{~km} \mathrm{~s}^{-1}\right)\end{array}$ & $\begin{array}{r}\log \sigma \\
\left(\mathrm{km} \mathrm{s}^{-1}\right)\end{array}$ & $\begin{array}{r}\log v_{\mathrm{m}} \\
\left(\mathrm{km} \mathrm{s}^{-1}\right)\end{array}$ & $\begin{array}{c}c z \\
\left(\mathrm{~km} \mathrm{~s}^{-1}\right)\end{array}$ & $(m-M)_{\mathrm{cin}}$ & $M_{\mathrm{B}}$ & FIR-B & $\log \frac{M_{\mathrm{HI}}}{L_{\mathrm{B}}}$ & $\log \frac{M_{\mathrm{mol}}}{L_{\mathrm{B}}}$ & Ref. & Notes \\
\hline 218 & 2.0 & 1.75 & 0.33 & 0.40 & 0.88 & 23.68 & 460 & 2.23 & 2.38 & 1053 & 31.01 & -19.93 & & -1.04 & $<-1.11$ & $b, d$ & \\
\hline 1525 & 5.9 & 1.73 & 0.93 & -0.15 & 0.41 & 24.49 & 221 & & 1.99 & 842 & 30.54 & -18.49 & & 0.02 & & $\mathrm{~d}$ & \\
\hline 2600 & 5.3 & 1.20 & 0.07 & & & 23.29 & & & 2.24 & 4452 & 34.04 & -20.87 & -1.51 & -0.79 & & d & \\
\hline 3043 & & 0.87 & 0.22 & & & 23.44 & 192 & & 1.98 & 1623 & 31.77 & -16.58 & & 0.46 & & $d$ & \\
\hline 3763 & 2.2 & 0.94 & 0.31 & & & 22.91 & 181 & & 1.93 & 4668 & 34.15 & -19.88 & -2.56 & -0.77 & & $\mathrm{~d}$ & \\
\hline 4785 & -0.1 & 1.29 & 0.22 & 0.06 & 0.66 & 23.47 & 244 & & 2.08 & 2133 & 32.46 & -19.55 & 0.03 & -0.83 & & $\mathrm{c}, \mathrm{d}$ & \\
\hline 4948 & 4.1 & 1.24 & 0.67 & & & 23.23 & 381 & & 2.23 & 2509 & 32.81 & -19.85 & -2.08 & -0.21 & & $d$ & \\
\hline 5139 & 5.3 & 1.56 & 0.08 & & 0.52 & 23.72 & 266 & & 2.30 & 2469 & 32.75 & -20.75 & 0.72 & -0.57 & & $\mathrm{~d}$ & \\
\hline 5194 & 5.7 & 1.18 & 0.82 & & & 24.13 & 161 & & 1.81 & 2415 & 32.72 & -18.47 & & -0.09 & & $d$ & \\
\hline 5232 & -2.0 & 1.18 & 0.35 & & & 24.49 & & & & 1930 & 32.20 & -17.70 & & & & & \\
\hline 5321 & 5.9 & 1.07 & 0.16 & & & 23.28 & 262 & & 2.18 & 4129 & 33.87 & -20.06 & & -0.76 & & $d$ & \\
\hline 5634 & 5.3 & 1.20 & 0.04 & & & 23.80 & 153 & & 2.17 & 3148 & 33.30 & -19.50 & & -0.47 & & $d$ & \\
\hline 5643 & 3.0 & 1.41 & 0.73 & & & 24.23 & 395 & & 2.26 & 2805 & 33.04 & -19.94 & -0.60 & -0.51 & & $d$ & \\
\hline 5998 & 5.9 & 0.88 & 0.18 & & & 22.10 & 212 & & 2.07 & 3183 & 33.27 & -19.43 & & -0.85 & & $d$ & \\
\hline 6275 & 3.1 & 1.39 & 0.24 & -0.18 & 0.55 & 23.57 & 322 & & 2.23 & 2987 & 33.16 & -20.42 & -0.41 & -0.27 & & $d$ & \\
\hline 6656 & 0.0 & 1.58 & 0.53 & & & 24.21 & 397 & & 2.26 & 1508 & 31.63 & -19.26 & & -0.41 & & $\mathrm{c}, \mathrm{d}$ & \\
\hline 6893 & 5.4 & 1.06 & 0.19 & & & 24.11 & 250 & & 2.13 & 4701 & 34.15 & -19.31 & & 0.12 & & $\mathrm{~d}$ & \\
\hline 6897 & 4.6 & 1.23 & 0.09 & -0.04 & 0.59 & 22.97 & 313 & & 2.35 & 4984 & 34.25 & -21.43 & -1.17 & -0.97 & & $d$ & \\
\hline 6993 & 1.1 & 1.37 & 0.04 & 0.33 & 0.83 & 23.10 & 124 & 2.13 & 2.10 & 1728 & 31.92 & -19.58 & 1.28 & -2.58 & & $\mathrm{~d}, \mathrm{e}$ & \\
\hline 7577 & 3.7 & 1.18 & 0.63 & & & 22.37 & & & & 3486 & 33.49 & -20.95 & & & & & \\
\hline 7826 & 5.4 & 0.96 & 0.04 & & & 23.76 & 96 & & 1.97 & 2379 & 32.69 & -17.79 & & 0.11 & & $d$ & \\
\hline 7952 & 6.6 & 1.21 & 0.06 & & & 24.57 & & & 1.96 & 3410 & 33.42 & -18.82 & & 0.13 & & $\mathrm{~d}$ & \\
\hline 8109 & & 0.93 & 0.35 & & & 23.50 & 248 & & 2.06 & 4516 & 34.06 & -19.23 & -1.79 & -0.36 & & $\mathrm{~d}$ & \\
\hline 8163 & 3.3 & 0.99 & 0.32 & & & 22.31 & & & & 4410 & 34.00 & -20.65 & -0.79 & & & $\mathrm{~d}$ & \\
\hline 8165 & 3.0 & 1.13 & 0.25 & & & 22.47 & 363 & & 2.29 & 4419 & 34.01 & -21.19 & -0.55 & -0.61 & & $\mathrm{~d}$ & \\
\hline 9126 & 6.5 & 1.20 & 0.14 & & & 23.45 & 118 & & 1.83 & 1385 & 31.36 & -17.78 & & -0.86 & & $\mathrm{~d}$ & \\
\hline 9988 & 1.0 & 1.38 & 0.05 & & & 24.27 & 181 & & 2.19 & 2630 & 32.81 & -19.36 & & -0.17 & & $\mathrm{~d}$ & \\
\hline 10789 & -1.8 & 1.00 & 0.39 & & & 22.08 & & & & 2596 & 32.78 & -19.69 & & & & & \\
\hline 10815 & 6.5 & 1.16 & 0.33 & & & 23.76 & & & 2.17 & 4502 & 34.01 & -20.12 & & -0.38 & & $\mathrm{~d}$ & \\
\hline 10942 & 3.0 & 1.25 & 0.24 & & & 23.77 & 290 & & 2.18 & 3040 & 33.15 & -19.80 & -0.83 & -0.11 & & $\mathrm{~d}$ & \\
\hline
\end{tabular}


Table 1. continued.

\begin{tabular}{|c|c|c|c|c|c|c|c|c|c|c|c|c|c|c|c|c|c|}
\hline PGC & $t$ & $\begin{array}{r}\log D_{25} \\
\quad\left(0^{\prime} 1\right)\end{array}$ & $\begin{array}{r}\log r_{25} \\
\left(0^{\prime} 1\right)\end{array}$ & $(U-B)_{0}$ & $(B-V)_{0}$ & $\begin{array}{c}\mu_{25} \\
\left(\mathrm{ma} / \operatorname{arcsec}^{2}\right)\end{array}$ & $\begin{array}{r}W_{20} \\
\left(\mathrm{~km} \mathrm{~s}^{-1}\right)\end{array}$ & $\begin{array}{r}\log \sigma \\
\left(\mathrm{km} \mathrm{s}^{-1}\right)\end{array}$ & $\begin{array}{r}\log v_{\mathrm{m}} \\
\left(\mathrm{km} \mathrm{s}^{-1}\right)\end{array}$ & $\begin{array}{c}c z \\
\left(\mathrm{~km} \mathrm{~s}^{-1}\right)\end{array}$ & $(m-M)_{\text {cin }}$ & $M_{\mathrm{B}}$ & FIR-B & $\log \frac{M_{\mathrm{HI}}}{L_{\mathrm{B}}}$ & $\log \frac{M_{\mathrm{mol}}}{L_{\mathrm{B}}}$ & Ref. & Notes \\
\hline 25467 & 0.1 & 1.04 & 0.28 & & & 23.05 & & & & 2992 & 33.22 & -19.21 & -0.31 & & & $\mathrm{~d}$ & \\
\hline 25985 & 2.2 & 0.89 & 0.22 & & & 23.45 & & & & 1934 & 32.33 & -17.14 & & & & & \\
\hline 26218 & -2.0 & 1.14 & 0.27 & & & 23.65 & & & & 1660 & 31.98 & -17.93 & -0.95 & & & $\mathrm{c}, \mathrm{d}$ & \\
\hline 26512 & 3.0 & 1.88 & 0.32 & 0.27 & 0.79 & 22.64 & 607 & 2.33 & 2.50 & 638 & 30.38 & -20.87 & 1.36 & -0.87 & -0.94 & $\mathrm{~b}, \mathrm{~d}$ & $\mathrm{CR}$ \\
\hline 26690 & 4.1 & 1.41 & 0.69 & & & 23.23 & 601 & & 2.46 & 4097 & 33.89 & -21.45 & & -0.37 & & $\mathrm{~d}$ & \\
\hline 26979 & 0.5 & 1.03 & 0.07 & -0.08 & 0.62 & 22.88 & 172 & & 1.98 & 1697 & 32.06 & -18.15 & -1.70 & -0.57 & & $\mathrm{~d}$ & \\
\hline 27077 & 4.0 & 2.10 & 0.36 & -0.02 & 0.57 & 23.16 & 384 & 2.01 & 2.37 & 556 & 29.76 & -20.90 & -0.24 & -0.94 & -1.02 & $\mathrm{~b}, \mathrm{~d}, \mathrm{f}$ & \\
\hline 27157 & -1.0 & 0.64 & 0.15 & & & 22.32 & & & & 1473 & 31.80 & -16.50 & & & & & \\
\hline 27311 & 2.8 & 0.89 & 0.43 & & & 23.50 & & & & 1654 & 32.00 & -16.71 & & & & & \\
\hline 27437 & 1.1 & 1.14 & 0.31 & & & 23.62 & 308 & & 2.18 & 4060 & 33.87 & -19.76 & & -0.51 & & $\mathrm{~d}$ & \\
\hline 27518 & 4.0 & 1.15 & 0.23 & & & 22.81 & 390 & & 2.34 & 4981 & 34.26 & -21.13 & -0.46 & -0.79 & & $\mathrm{~d}$ & \\
\hline 27792 & 4.3 & 1.00 & 0.09 & & & 23.29 & & & 1.97 & 1466 & 31.83 & -17.36 & & 0.09 & & $\mathrm{~d}$ & \\
\hline 27796 & 3.1 & 1.24 & 0.46 & & & 23.45 & & & 2.34 & 4940 & 34.28 & -20.81 & -0.05 & -0.44 & & $\mathrm{~d}$ & \\
\hline 27968 & 6.5 & 1.10 & 0.30 & & & 24.89 & 191 & & 1.95 & 3088 & 33.22 & -17.66 & & 0.42 & & $\mathrm{~d}$ & \\
\hline 28145 & 1.5 & 0.94 & 0.47 & & & 22.76 & & & & 4679 & 34.19 & -19.86 & -1.90 & & & $\mathrm{~d}$ & \\
\hline 28259 & -1.7 & 0.92 & 0.07 & & & 23.21 & 198 & & 2.14 & 1524 & 31.86 & -17.10 & & -1.11 & & $\mathrm{~d}$ & $\mathrm{CR}$ \\
\hline 28401 & 3.6 & 1.13 & 0.04 & & & 23.75 & 60 & & 1.55 & 3365 & 33.56 & -19.88 & -0.48 & -0.41 & & $\mathrm{~d}$ & \\
\hline 28424 & -1.9 & 1.25 & 0.04 & 0.09 & 0.63 & 23.29 & 241 & 1.91 & 2.28 & 1538 & 31.86 & -18.67 & -0.81 & -1.43 & -0.75 & $\mathrm{~b}, \mathrm{c}, \mathrm{d}, \mathrm{e}$ & \\
\hline 28485 & 5.3 & 1.57 & 0.19 & & 0.69 & 23.54 & 294 & & 2.19 & 1412 & 31.60 & -19.78 & 0.38 & -0.73 & & $\mathrm{~d}$ & \\
\hline 28672 & 3.1 & 1.24 & 0.50 & & & 22.45 & 312 & & 2.12 & 2986 & 33.31 & -20.78 & 0.12 & -1.14 & & $\mathrm{~d}$ & \\
\hline 28758 & 0.8 & 0.84 & 0.22 & & & 22.91 & 202 & & 1.93 & 1486 & 31.82 & -16.90 & & -0.24 & & $\mathrm{~d}$ & \\
\hline 29009 & 1.1 & 1.25 & 0.15 & & & 23.11 & 261 & & 2.18 & 2406 & 32.73 & -19.75 & -2.31 & -0.63 & & $\mathrm{~d}$ & \\
\hline 29177 & -3.1 & 0.74 & 0.07 & -0.27 & 0.53 & 22.25 & & & & 2605 & 33.04 & -18.33 & -1.68 & -0.27 & & $\mathrm{~d}$ & \\
\hline 29198 & & 0.63 & 0.19 & & & 23.15 & & & & 1112 & 31.02 & -14.87 & & & & & \\
\hline 29347 & -2.4 & 0.82 & 0.03 & -0.14 & 0.39 & 22.54 & 115 & & 2.03 & 1362 & 31.62 & -17.04 & & -0.97 & & $\mathrm{~d}$ & \\
\hline 29715 & 3.0 & 1.26 & 0.46 & & & 24.52 & 442 & & 2.33 & 4754 & 34.21 & -19.89 & -0.95 & 0.01 & & $\mathrm{~d}$ & \\
\hline 30010 & 3.0 & 1.19 & 0.12 & 0.03 & 0.59 & 22.47 & 276 & & 2.19 & 1308 & 31.49 & -18.77 & -2.03 & -1.06 & & $\mathrm{~d}$ & \\
\hline 30197 & 5.2 & 1.89 & 0.48 & -0.12 & 0.43 & 23.39 & 322 & 1.81 & 2.19 & 663 & 30.43 & -20.21 & 0.69 & -0.37 & & $\mathrm{~d}$ & \\
\hline 30310 & 4.0 & 0.92 & 0.13 & & & 24.18 & & & 1.93 & 2906 & 33.12 & -17.49 & & 0.13 & & $\mathrm{~d}$ & \\
\hline 30569 & 5.9 & 1.27 & 0.32 & & & 23.73 & 259 & & 2.11 & 2127 & 32.64 & -18.97 & -0.31 & -0.09 & & $\mathrm{~d}$ & \\
\hline 30858 & 4.5 & 0.90 & 0.15 & & & 23.59 & & & & 2502 & 32.96 & -17.63 & & & & & \\
\hline 30895 & 4.0 & 1.63 & 0.40 & 0.01 & 0.58 & 23.82 & 431 & 2.06 & 2.31 & 1352 & 31.62 & -19.73 & & -0.44 & & $\mathrm{~d}$ & \\
\hline 31304 & -0.9 & 1.08 & 0.11 & & & 22.89 & & & & 957 & 30.63 & -17.12 & & & & & \\
\hline
\end{tabular}

. 
Table 1. continued.

\begin{tabular}{|c|c|c|c|c|c|c|c|c|c|c|c|c|c|c|c|c|c|}
\hline PGC & $t$ & $\begin{array}{r}\log D_{25} \\
(0.1)\end{array}$ & $\begin{array}{r}\log r_{25} \\
\left(0^{\prime} 1\right)\end{array}$ & $(U-B)_{0}$ & $(B-V)_{0}$ & $\begin{array}{c}\mu_{25} \\
\left(\mathrm{ma} / \operatorname{arcsec}^{2}\right)\end{array}$ & $\begin{array}{r}W_{20} \\
\left(\mathrm{~km} \mathrm{~s}^{-1}\right)\end{array}$ & $\begin{array}{r}\log \sigma \\
\left(\mathrm{km} \mathrm{s}^{-1}\right)\end{array}$ & $\begin{array}{r}\log v_{\mathrm{m}} \\
\left(\mathrm{km} \mathrm{s}^{-1}\right)\end{array}$ & $\begin{array}{c}c z \\
\left(\mathrm{~km} \mathrm{~s}^{-1}\right)\end{array}$ & $(m-M)_{\text {cin }}$ & $\mathrm{M}_{\mathrm{B}}$ & FIR-B & $\log \frac{M_{\mathrm{HI}}}{L_{\mathrm{B}}}$ & $\log \frac{M_{\mathrm{mol}}}{L_{\mathrm{B}}}$ & Ref. & Notes \\
\hline 31472 & -1.9 & 1.21 & 0.28 & & & 23.42 & & 2.20 & & 3054 & 33.24 & -19.76 & & $<-1.57$ & & $\mathrm{c}, \mathrm{e}$ & \\
\hline 31601 & & 0.65 & 0.00 & & & 21.95 & 118 & & & 1699 & 32.22 & -17.34 & & -0.61 & & $\mathrm{~d}$ & \\
\hline 31650 & 4.0 & 1.44 & 0.02 & -0.45 & 0.32 & 21.98 & 233 & 2.05 & 2.24 & 988 & 31.19 & -20.25 & -1.45 & -0.63 & -1.50 & $\mathrm{~b}, \mathrm{~d}, \mathrm{~g}$ & ARP217 \\
\hline 31883 & 5.1 & 1.61 & 0.22 & -0.07 & 0.51 & 23.47 & 352 & & 2.26 & 1301 & 31.44 & -19.87 & 0.08 & -0.13 & -1.11 & $\mathrm{~b}, \mathrm{~d}$ & \\
\hline 31945 & -2.0 & 1.11 & 0.49 & -0.14 & 0.60 & 23.86 & 121 & & 1.63 & 1321 & 31.52 & -17.01 & -0.80 & -0.53 & & $\mathrm{~d}$ & \\
\hline 32183 & 5.2 & 1.86 & 0.20 & -0.24 & 0.41 & 23.92 & 255 & & 2.14 & 1012 & 31.28 & -20.42 & 0.40 & -0.18 & -1.21 & $\mathrm{~b}, \mathrm{~d}$ & \\
\hline 32364 & 0.1 & 0.98 & 0.36 & & & 22.43 & 167 & & 1.82 & 712 & 30.12 & -16.36 & & -0.81 & & $\mathrm{~d}$ & \\
\hline 32543 & -1.8 & 1.26 & 0.36 & & & 22.98 & 186 & & 1.86 & 646 & 30.27 & -17.39 & 0.21 & -0.20 & $<-0.82$ & $\mathrm{~b}, \mathrm{c}, \mathrm{d}$ & \\
\hline 32719 & 4.0 & 1.48 & 0.38 & & & 23.54 & 298 & & 2.15 & 1258 & 31.70 & -19.66 & 0.56 & -0.32 & & $\mathrm{~d}$ & \\
\hline 33140 & 3.1 & 1.37 & 0.03 & & & 23.18 & 142 & & 2.16 & 1434 & 31.66 & -19.18 & & 0.07 & & $\mathrm{~d}$ & \\
\hline 33375 & 0.0 & 0.87 & 0.06 & & & 22.53 & & & & 1549 & 32.03 & -17.65 & & & & & \\
\hline 33604 & 0.1 & 1.16 & 0.52 & & & 22.41 & & & & 1344 & 31.50 & -18.63 & & & & & \\
\hline 33726 & & 0.98 & 0.07 & & & 23.61 & 100 & & 1.84 & 1228 & 31.59 & -16.65 & & 0.23 & & $\mathrm{~d}, \mathrm{~g}$ & \\
\hline 34353 & 1.1 & 1.56 & 0.59 & & & 24.91 & 214 & & 1.95 & 719 & 30.57 & -17.16 & -0.60 & 0.57 & & $\mathrm{~d}$ & \\
\hline 34692 & 4.4 & 1.07 & 0.40 & & 0.39 & 22.01 & 158 & & 1.83 & 1314 & 31.76 & -18.84 & -0.04 & -0.56 & & $\mathrm{~d}$ & \\
\hline 34767 & 5.2 & 1.69 & 0.08 & & 0.54 & 23.11 & 124 & & 1.92 & 1159 & 31.49 & -20.62 & -0.17 & -0.68 & -0.55 & $\mathrm{~b}, \mathrm{~d}$ & ARP27 \\
\hline 34836 & 4.6 & 1.57 & 0.28 & -0.10 & 0.55 & 23.08 & 532 & & 2.54 & 4256 & 33.97 & -22.57 & & -0.72 & & $\mathrm{~d}$ & \\
\hline 34908 & -2.0 & 1.08 & 0.21 & & & 23.19 & & & & 2050 & 32.52 & -18.57 & & & & $\mathrm{c}$ & \\
\hline 34935 & 4.9 & 1.17 & 0.18 & 0.01 & 0.58 & 21.51 & 310 & & 2.26 & 1480 & 31.75 & -19.89 & -1.07 & -0.99 & & $\mathrm{~d}$ & \\
\hline 34967 & 7.8 & 0.95 & 0.53 & & & 22.13 & 269 & & 2.06 & 2607 & 32.86 & -19.30 & -0.75 & -0.45 & & $\mathrm{~d}$ & \\
\hline 35025 & 4.7 & 1.17 & 0.24 & & & 22.71 & 242 & & 2.09 & 1570 & 32.10 & -19.01 & 0.11 & -1.00 & & $\mathrm{~d}$ & \\
\hline 35164 & 3.0 & 1.80 & 0.25 & & & 23.30 & 429 & 2.08 & 2.37 & 767 & 30.70 & -20.16 & & -0.87 & -0.64 & $\mathrm{~b}, \mathrm{~d}$ & \\
\hline 35225 & & 1.04 & 0.38 & & & 23.43 & & & 1.30 & 1039 & 30.96 & -16.71 & & -0.80 & & $\mathrm{~d}$ & \\
\hline 35266 & 0.0 & 1.25 & 0.17 & 0.01 & 0.71 & 23.16 & 295 & & 2.21 & 1512 & 32.02 & -18.90 & -1.46 & -1.17 & & $\mathrm{c}, \mathrm{d}$ & \\
\hline 35314 & 3.1 & 1.50 & 0.66 & & & 22.77 & 404 & & 2.28 & 1724 & 32.02 & -20.50 & 1.22 & -0.82 & & $\mathrm{~d}$ & \\
\hline 35440 & 2.4 & 1.66 & 0.39 & 0.04 & 0.67 & 23.30 & 354 & & 2.23 & 1017 & 30.92 & -19.80 & 0.35 & -0.63 & & $\mathrm{~d}$ & \\
\hline 35608 & 2.1 & 1.22 & 0.38 & & & 23.33 & 223 & & 1.99 & 1913 & 32.48 & -19.15 & -0.06 & -1.00 & & $\mathrm{~d}$ & \\
\hline 35676 & 5.1 & 1.78 & 0.17 & & 0.44 & 23.22 & 288 & 1.86 & 2.16 & 857 & 30.92 & -20.39 & 0.81 & -0.53 & -0.95 & $\mathrm{~b}, \mathrm{~d}$ & \\
\hline 35955 & -2.0 & 1.06 & 0.02 & & & 22.68 & & & & 1269 & 31.68 & -18.13 & & & & $\mathrm{c}$ & \\
\hline 36037 & -3.3 & 0.99 & 0.00 & & & 22.88 & & & & 1337 & 31.78 & -17.71 & & & & $\mathrm{c}$ & \\
\hline 36043 & -2.0 & 1.08 & 0.10 & & & 23.28 & 146 & & 1.87 & 976 & 30.87 & -16.88 & -1.14 & -0.80 & & $\mathrm{c}, \mathrm{d}$ & \\
\hline 36211 & -3.8 & 0.86 & 0.28 & & & 23.61 & 164 & & 1.81 & 1837 & 32.26 & -16.87 & & -0.10 & & $\mathrm{~d}$ & \\
\hline 36215 & 3.8 & 1.11 & 0.15 & & & 22.55 & & & & 1255 & 31.66 & -18.46 & & & & & \\
\hline
\end{tabular}


Table 1. continued.

\begin{tabular}{|c|c|c|c|c|c|c|c|c|c|c|c|c|c|c|c|c|c|}
\hline PGC & $t$ & $\begin{array}{r}\log D_{25} \\
\left(0^{\prime} 1\right)\end{array}$ & $\begin{array}{r}\log r_{25} \\
(0,1)\end{array}$ & $(U-B)_{0}$ & $(B-V)_{0}$ & $\begin{array}{c}\mu_{25} \\
\left(\mathrm{ma} / \operatorname{arcsec}^{2}\right)\end{array}$ & $\begin{array}{r}W_{20} \\
\left(\mathrm{~km} \mathrm{~s}^{-1}\right)\end{array}$ & $\begin{array}{r}\log \sigma \\
\left(\mathrm{km} \mathrm{s}^{-1}\right)\end{array}$ & $\begin{array}{r}\log v_{\mathrm{m}} \\
\left(\mathrm{km} \mathrm{s}^{-1}\right)\end{array}$ & $\begin{array}{c}c z \\
\left(\mathrm{~km} \mathrm{~s}^{-1}\right)\end{array}$ & $(m-M)_{\mathrm{cin}}$ & $M_{\mathrm{B}}$ & FIR-B & $\log \frac{M_{\mathrm{HI}}}{L_{\mathrm{B}}}$ & $\log \frac{M_{\mathrm{mol}}}{L_{\mathrm{B}}}$ & Ref. & Notes \\
\hline 36266 & 3.3 & 1.30 & 0.29 & -0.14 & 0.50 & 21.99 & 316 & & 2.20 & 1466 & 31.85 & -20.13 & & -0.67 & & $\mathrm{~d}$ & \\
\hline 36686 & -2.0 & 1.02 & 0.09 & -0.24 & 0.43 & 22.37 & 123 & & 1.83 & 755 & 30.73 & -17.28 & -0.56 & -0.67 & & $\mathrm{c}, \mathrm{d}$ & \\
\hline 36776 & 3.2 & 1.01 & 0.00 & & & 22.79 & 172 & & 3.10 & 3572 & 33.69 & -19.88 & & -1.13 & & $\mathrm{~d}$ & \\
\hline 36930 & 5.8 & 1.42 & 0.78 & & & 23.83 & 177 & & 1.86 & 848 & 30.86 & -17.81 & & -0.24 & & $\mathrm{~d}$ & \\
\hline 37213 & 5.0 & 0.82 & 0.10 & & 0.20 & 22.35 & & & 1.68 & 1055 & 30.90 & -16.46 & & -0.29 & & $\mathrm{~d}$ & \\
\hline 37235 & -2.0 & 1.55 & 0.15 & 0.41 & 0.87 & 22.71 & 258 & 2.12 & 2.13 & 921 & 30.99 & -19.87 & & -1.40 & & $\mathrm{~d}, \mathrm{e}$ & $\mathrm{CR}$ \\
\hline 37244 & -3.1 & 1.05 & 0.13 & & & 23.49 & & 2.05 & & 3650 & 33.67 & -19.31 & & & & & \\
\hline 37290 & 4.0 & 1.43 & 0.23 & & 0.38 & 22.15 & 281 & & 2.13 & 800 & 30.82 & -19.59 & -0.78 & -0.72 & -1.18 & $\mathrm{~b}, \mathrm{~d}$ & \\
\hline 37352 & 7.1 & 1.20 & 0.50 & & & 23.00 & 246 & & 2.02 & 2384 & 32.67 & -19.43 & & -0.50 & & $\mathrm{~d}$ & \\
\hline 37444 & 5.9 & 1.40 & 0.06 & & & 24.23 & 187 & & 2.20 & 1892 & 32.19 & -18.80 & & -0.10 & & $\mathrm{~d}$ & \\
\hline 37574 & -2.0 & 1.02 & 0.10 & & & 23.61 & & & & 3309 & 33.48 & -18.82 & & & & & \\
\hline 37584 & 6.7 & 1.52 & 0.08 & & & 24.09 & 132 & & 1.96 & 778 & 30.78 & -18.14 & & -0.16 & & $\mathrm{~d}$ & \\
\hline 37795 & -2.4 & 0.90 & 0.00 & & & 22.90 & & & & 3139 & 33.37 & -18.83 & & & & & \\
\hline 37838 & 4.3 & 0.94 & 0.33 & & & 23.32 & 121 & & 1.71 & 622 & 30.25 & -15.39 & & -0.43 & & $\mathrm{~d}$ & \\
\hline 37928 & 3.1 & 1.40 & 0.04 & & & 23.70 & 95 & & 1.86 & 932 & 30.81 & -17.96 & 0.75 & -1.05 & & $\mathrm{~d}$ & \\
\hline 38068 & 4.0 & 1.71 & 0.06 & & 0.62 & 23.09 & 284 & 1.94 & 2.39 & 710 & 30.60 & -19.84 & -0.07 & -0.86 & -0.85 & $b, d$ & \\
\hline 38150 & 5.3 & 1.61 & 0.39 & -0.03 & 0.66 & 22.99 & 301 & 1.99 & 2.18 & 769 & 30.63 & -19.45 & 0.46 & -0.97 & -1.20 & $\mathrm{~d}, \mathrm{f}$ & \\
\hline 38277 & 1.3 & 0.78 & 0.07 & & & 22.68 & 175 & & 2.11 & 581 & 30.13 & -15.17 & & -0.70 & & $\mathrm{~d}$ & \\
\hline 38286 & 2.5 & 0.79 & 0.26 & & & 23.22 & & & & 538 & 29.97 & -14.49 & & & & & \\
\hline 38392 & 3.0 & 1.49 & 0.25 & & & 22.81 & 328 & & 2.23 & 843 & 30.94 & -19.38 & -2.52 & -1.20 & -0.57 & $\mathrm{~b}, \mathrm{~d}$ & \\
\hline 38527 & -1.3 & 1.50 & 0.25 & 0.22 & 0.76 & 23.64 & & 1.70 & & 1656 & 31.96 & -19.64 & & $<-2.15$ & & $\mathrm{c}, \mathrm{d}, \mathrm{e}$ & \\
\hline 38582 & 2.0 & 1.06 & 0.19 & & & 23.03 & 153 & & 1.89 & 946 & 31.08 & -17.09 & & -1.16 & & $\mathrm{~d}$ & \\
\hline 38800 & -1.7 & 0.82 & 0.05 & & & 22.94 & & & & 1078 & 31.12 & -16.22 & & & & & \\
\hline 38802 & 3.0 & 1.25 & 0.09 & & & 23.28 & 315 & & 2.36 & 2460 & 32.83 & -19.66 & -0.14 & -0.61 & -0.91 & $\mathrm{a}, \mathrm{d}, \mathrm{e}$ & \\
\hline 38964 & 2.1 & 1.19 & 0.46 & & & 22.37 & 416 & & 2.30 & 2083 & 32.43 & -19.73 & -1.26 & -1.24 & & $\mathrm{~d}$ & \\
\hline 39251 & -0.8 & 1.28 & 0.40 & 0.30 & 0.85 & 23.06 & & & & 2072 & 32.41 & -19.52 & & $<-1.60$ & & $\mathrm{c}, \mathrm{e}$ & \\
\hline 39393 & 4.0 & 1.32 & 0.19 & & 0.80 & 22.51 & 259 & & 2.16 & 864 & 30.69 & -18.65 & -0.34 & -1.37 & -1.30 & $\mathrm{~b}, \mathrm{~d}$ & \\
\hline 39483 & 5.5 & 1.18 & 0.13 & & 0.63 & 23.47 & 127 & & 1.86 & 733 & 30.27 & -16.53 & & -0.37 & & $\mathrm{~d}$ & \\
\hline 39525 & 1.0 & 0.95 & 0.09 & -0.01 & 0.62 & 22.34 & & & & 3875 & 33.81 & -20.09 & -1.88 & & & $\mathrm{~d}$ & \\
\hline 39681 & 4.8 & 1.02 & 0.19 & & & 24.18 & & & & 925 & 30.85 & -15.57 & & & & & \\
\hline 40330 & 5.0 & 1.47 & 0.40 & & & 24.48 & 211 & & 1.97 & 1248 & 31.54 & -18.20 & 0.32 & -0.13 & -1.42 & $\mathrm{~d}, \mathrm{f}$ & \\
\hline 40396 & 1.0 & 1.32 & 0.02 & -0.04 & 0.62 & 22.82 & 134 & & 2.19 & 1028 & 31.22 & -18.85 & -1.20 & -1.12 & & $\mathrm{c}, \mathrm{d}$ & \\
\hline 40475 & 1.0 & 1.11 & 0.11 & -0.28 & 0.40 & 22.68 & 141 & & 1.91 & 2513 & 32.98 & -19.70 & -1.02 & -0.82 & & $\mathrm{~d}$ & \\
\hline
\end{tabular}


Table 1. continued.

\begin{tabular}{|c|c|c|c|c|c|c|c|c|c|c|c|c|c|c|c|c|c|}
\hline PGC & $t$ & $\begin{array}{r}\log D_{25} \\
(0.1)\end{array}$ & $\begin{array}{r}\log r_{25} \\
(0.1)\end{array}$ & $(U-B)_{0}$ & $(B-V)_{0}$ & $\begin{array}{c}\mu_{25} \\
\left(\mathrm{ma} / \operatorname{arcsec}^{2}\right)\end{array}$ & $\begin{array}{r}W_{20} \\
\left(\mathrm{~km} \mathrm{~s}^{-1}\right)\end{array}$ & $\begin{array}{r}\log \sigma \\
\left(\mathrm{km} \mathrm{s}^{-1}\right)\end{array}$ & $\begin{array}{r}\log v_{\mathrm{m}} \\
\left(\mathrm{km} \mathrm{s}^{-1}\right)\end{array}$ & $\begin{array}{c}c z \\
\left(\mathrm{~km} \mathrm{~s}^{-1}\right)\end{array}$ & $(m-M)_{\mathrm{cin}}$ & $M_{\mathrm{B}}$ & FIR-B & $\log \frac{M_{\mathrm{HI}}}{L_{\mathrm{B}}}$ & $\log \frac{M_{\mathrm{mol}}}{L_{\mathrm{B}}}$ & Ref. & Notes \\
\hline 40490 & 1.0 & 1.40 & 0.03 & 0.43 & 0.91 & 23.22 & 350 & 2.28 & 2.64 & 2551 & 32.85 & -20.46 & & -0.79 & & $\mathrm{~d}, \mathrm{e}$ & \\
\hline 40500 & -3.0 & 1.05 & 0.00 & & & 23.00 & & & & 1358 & 31.83 & -17.96 & & & & $\mathrm{c}$ & \\
\hline 40715 & 3.2 & 1.16 & 0.05 & & & 22.67 & 136 & & 2.05 & 2295 & 32.62 & -19.55 & -1.16 & -1.01 & & d & \\
\hline 40775 & 4.7 & 1.30 & 0.32 & & & 22.51 & 208 & & 1.98 & 1689 & 31.97 & -19.72 & -0.37 & -0.81 & & d & \\
\hline 41013 & 5.9 & 1.05 & 0.10 & & & 22.68 & 134 & & 1.91 & 1702 & 32.07 & -18.51 & 0.09 & -1.06 & & d & \\
\hline 41317 & 5.1 & 1.33 & 0.30 & & 0.61 & 23.06 & 340 & & 2.23 & 2443 & 32.76 & -20.14 & 0.07 & -0.66 & & $d$ & \\
\hline 41436 & 1.1 & 1.21 & 0.16 & -0.10 & 0.53 & 22.72 & 332 & & 2.29 & 3117 & 33.41 & -20.55 & -1.18 & -0.96 & & d & \\
\hline 41652 & -2.9 & 1.12 & 0.09 & & & 22.63 & & 1.96 & & 979 & 30.96 & -17.84 & & & & $\mathrm{c}$ & \\
\hline 42174 & 1.6 & 1.30 & 0.15 & & & 22.90 & 196 & & 2.07 & 1036 & 30.97 & -18.39 & -0.02 & -1.89 & $<-1.38$ & $\mathrm{~b}, \mathrm{~d}, \mathrm{a}$ & \\
\hline 42396 & 3.4 & 1.22 & 0.20 & & & 22.43 & 153 & & 1.89 & 632 & 30.12 & -17.66 & 0.47 & -0.96 & & $\mathrm{a}, \mathrm{d}$ & \\
\hline 42497 & -1.3 & 0.79 & 0.24 & & & 23.82 & & & & 718 & 30.22 & -14.18 & & & & & \\
\hline 42605 & -3.4 & 0.98 & 0.32 & & & 23.84 & & & & 1032 & 31.00 & -15.84 & & & & & \\
\hline 42833 & 5.2 & 1.60 & 0.18 & & 0.50 & 22.84 & 384 & & 2.34 & 804 & 30.59 & -19.58 & 0.08 & -0.63 & -1.07 & $b, d$ & ARP189, VV56 \\
\hline 43106 & 6.0 & 1.30 & 0.13 & & & 23.90 & 134 & & 1.89 & 836 & 30.55 & -16.98 & & -0.40 & & d & \\
\hline 43121 & -3.4 & 0.79 & 0.15 & & & 23.24 & & & & 830 & 30.75 & -15.24 & & & & $\mathrm{~d}$ & \\
\hline 43254 & 1.4 & 1.58 & 0.24 & 0.37 & 0.83 & 22.99 & 417 & 2.24 & 2.40 & 1003 & 30.94 & -19.64 & & -0.75 & $<-1.45$ & $\mathrm{a}, \mathrm{b}, \mathrm{d}, \mathrm{e}$ & \\
\hline 43375 & -0.8 & 1.69 & 0.49 & 0.29 & 0.78 & 23.95 & 333 & & 2.20 & 1324 & 31.56 & -19.84 & -0.62 & -2.07 & -1.03 & $\mathrm{~b}, \mathrm{c}, \mathrm{d}, \mathrm{e}$ & \\
\hline 43671 & -1.6 & 1.74 & 0.27 & 0.36 & 0.82 & 23.52 & 80 & 2.34 & 1.48 & 1395 & 31.55 & -20.59 & 0.85 & -2.68 & $<-1.63$ & $\mathrm{~b}, \mathrm{c}, \mathrm{d}, \mathrm{e}, \mathrm{g}$ & \\
\hline 43931 & 3.0 & 1.22 & 0.15 & & & 22.12 & 308 & & 2.28 & 862 & 30.97 & -18.72 & -1.10 & -0.79 & & d & \\
\hline 44370 & 6.2 & 0.97 & 0.17 & -0.23 & 0.45 & 23.41 & & & 1.96 & 2526 & 32.93 & -18.15 & & -0.53 & $<-0.10$ & $\mathrm{a}, \mathrm{d}$ & \\
\hline 44797 & 5.2 & 1.34 & 0.02 & & 0.50 & 22.35 & 152 & & 2.28 & 968 & 30.82 & -19.01 & -0.62 & -0.82 & & $\mathrm{~d}$ & \\
\hline 44961 & 6.4 & 1.50 & 0.77 & & & 24.32 & 168 & & 1.89 & 669 & 30.62 & -17.44 & & 0.27 & & $d$ & \\
\hline 45836 & 4.4 & 1.22 & 0.13 & & & 23.34 & 263 & & 2.21 & 2612 & 32.99 & -19.53 & -0.80 & -0.41 & -0.86 & $\mathrm{a}, \mathrm{d}$ & \\
\hline 45879 & 4.0 & 1.07 & 0.68 & & & 23.44 & & & & 3142 & 33.45 & -19.05 & -0.84 & & & $\mathrm{~d}$ & \\
\hline 45883 & 3.8 & 1.47 & 0.05 & & & 24.11 & 243 & & 2.33 & 3360 & 33.47 & -20.60 & -1.55 & -0.27 & & $d$ & \\
\hline 46934 & 3.3 & 1.31 & 0.07 & & & 23.71 & 226 & & 2.25 & 1225 & 31.59 & -18.21 & -2.27 & -0.30 & & d & \\
\hline 47482 & 3.2 & 0.99 & 0.10 & 0.04 & 0.69 & 22.84 & 298 & & 2.30 & 4130 & 33.92 & -19.89 & -0.25 & -1.00 & & $d$ & \\
\hline 47577 & 5.9 & 1.06 & 0.17 & & & 23.06 & 121 & & 1.79 & 1020 & 31.10 & -17.16 & -0.43 & -0.62 & & $d$ & \\
\hline 47938 & -1.6 & 0.90 & 0.49 & & & 24.08 & 122 & & 1.58 & 853 & 30.85 & -14.99 & & 0.30 & & d & \\
\hline 48521 & -1.9 & 1.44 & 0.10 & 0.33 & 0.82 & 23.47 & & 1.83 & & 1108 & 31.37 & -18.90 & & $<-1.76$ & & $\mathrm{c}, \mathrm{e}$ & \\
\hline
\end{tabular}


Table 1. continued.

\begin{tabular}{|c|c|c|c|c|c|c|c|c|c|c|c|c|c|c|c|c|c|}
\hline PGC & $t$ & $\begin{array}{r}\log D_{25} \\
\left(0^{\prime} 1\right)\end{array}$ & $\begin{array}{r}\log r_{25} \\
\left(0^{\prime} 1\right)\end{array}$ & $(U-B)_{0}$ & $(B-V)_{0}$ & $\begin{array}{c}\mu_{25} \\
\left(\mathrm{ma} / \operatorname{arcsec}^{2}\right)\end{array}$ & $\begin{array}{r}W_{20} \\
\left(\mathrm{~km} \mathrm{~s}^{-1}\right)\end{array}$ & $\begin{array}{r}\log \sigma \\
\left(\mathrm{km} \mathrm{s}^{-1}\right)\end{array}$ & $\begin{array}{r}\log v_{\mathrm{m}} \\
\left(\mathrm{km} \mathrm{s}^{-1}\right)\end{array}$ & $\begin{array}{c}c z \\
\left(\mathrm{~km} \mathrm{~s}^{-1}\right)\end{array}$ & $(m-M)_{\text {cin }}$ & $M_{\mathrm{B}}$ & FIR-B & $\log \frac{M_{\mathrm{HI}}}{L_{\mathrm{B}}}$ & $\log \frac{M_{\mathrm{mol}}}{L_{\mathrm{B}}}$ & Ref. & Notes \\
\hline 48959 & 5.3 & 1.54 & 0.19 & & & 23.83 & 231 & & 2.09 & 1172 & 31.25 & -18.94 & 0.41 & -0.63 & & $\mathrm{~d}$ & \\
\hline 49112 & 5.2 & 1.48 & 0.30 & & & 24.27 & 307 & & 2.19 & 2614 & 33.04 & -19.89 & -0.30 & -0.16 & & $\mathrm{~d}$ & \\
\hline 49308 & 5.1 & 1.59 & 0.13 & & & 24.20 & 240 & & 2.16 & 1382 & 31.56 & -19.22 & 0.59 & -0.43 & & $\mathrm{~d}$ & \\
\hline 49451 & & 0.81 & 0.25 & & & 21.70 & & & & 1716 & 32.27 & -18.41 & -1.68 & & & $\mathrm{~d}$ & \\
\hline 49927 & -1.0 & 0.83 & 0.03 & & & 22.78 & & & & 2684 & 33.09 & -18.27 & & & & & \\
\hline 49956 & -0.1 & 1.05 & 0.15 & & & 23.39 & 346 & & 2.31 & 3652 & 33.71 & -19.37 & & -0.07 & & $\mathrm{~d}$ & \\
\hline 50116 & 3.1 & 1.03 & 0.56 & & & 22.18 & & & & 3830 & 33.81 & -20.46 & & & & & \\
\hline 50144 & -2.0 & 1.15 & 0.00 & & & 25.31 & & & & 1224 & 31.35 & -15.70 & & & & & \\
\hline 50198 & 1.3 & 0.74 & 0.21 & & & 23.00 & & & & 4334 & 34.06 & -18.57 & -1.97 & & & $\mathrm{~d}$ & \\
\hline 50479 & -1.4 & 1.19 & 0.35 & & & 23.91 & & & & 1531 & 31.78 & -17.73 & & & & & \\
\hline 50745 & 1.2 & 0.99 & 0.12 & & & 21.91 & & & & 4277 & 33.99 & -20.88 & -0.05 & & & $\mathrm{~d}$ & \\
\hline 50889 & 3.1 & 1.27 & 0.66 & & & 23.26 & 451 & & 2.31 & 4574 & 34.14 & -20.99 & -0.86 & -0.36 & & $\mathrm{~d}$ & \\
\hline 51091 & 2.8 & 1.07 & 0.40 & & & 23.28 & 390 & & 2.28 & 4280 & 34.03 & -19.86 & -0.76 & -0.20 & & $\mathrm{~d}$ & \\
\hline 51895 & -1.9 & 1.10 & 0.38 & & & 23.80 & 238 & & 1.98 & 1454 & 31.71 & -17.24 & -2.87 & 0.19 & & $\mathrm{c}, \mathrm{d}$ & \\
\hline 51951 & 0.1 & 0.60 & 0.03 & & & 21.66 & & & & 4281 & 34.03 & -19.25 & -1.20 & & & $\mathrm{~d}$ & \\
\hline 52273 & 5.4 & 1.52 & 0.52 & & & 23.02 & 310 & & 2.15 & 1753 & 32.09 & -20.50 & -0.64 & -0.69 & & $\mathrm{~d}$ & \\
\hline 52488 & 3.0 & 1.07 & 0.41 & & & 24.16 & & & 2.04 & 4605 & 34.14 & -19.19 & & -0.22 & & $\mathrm{~d}$ & \\
\hline 52607 & 5.9 & 1.18 & 0.30 & & & 22.99 & 255 & & 2.09 & 3284 & 33.53 & -20.19 & 0.18 & -1.15 & & $\mathrm{~d}$ & \\
\hline 52636 & -3.8 & 0.90 & 0.00 & & & 23.93 & & & & 3693 & 33.72 & -18.30 & & & & & \\
\hline 52741 & -1.9 & 0.91 & 0.07 & & & 22.90 & & 2.07 & & 1693 & 32.18 & -17.68 & -0.42 & & & $\mathrm{~d}$ & \\
\hline 52887 & 3.8 & 1.17 & 0.13 & & & 24.20 & 189 & & 2.06 & 1781 & 32.18 & -17.70 & & 0.00 & & $\mathrm{~d}$ & \\
\hline 53641 & 2.7 & 0.90 & 0.32 & & & 22.10 & 95 & & 1.56 & 608 & 30.45 & -16.60 & & -0.40 & & $\mathrm{~d}$ & \\
\hline 54265 & 0.9 & 1.12 & 0.49 & & & 23.61 & 453 & & 2.33 & 3374 & 33.59 & -19.41 & & -0.29 & & $\mathrm{~d}$ & \\
\hline 54909 & 5.9 & 0.92 & 0.54 & & & 23.80 & 181 & & 1.88 & 1830 & 32.20 & -16.84 & & -0.12 & & $\mathrm{~d}$ & \\
\hline 55419 & 4.7 & 1.52 & 0.16 & -0.20 & 0.46 & 24.04 & 215 & & 2.08 & 655 & 30.63 & -17.95 & -0.42 & -0.07 & & $\mathrm{~d}$ & \\
\hline 55802 & 6.0 & 0.96 & 0.03 & & & 22.27 & & & 2.31 & 2864 & 33.27 & -19.64 & -0.63 & -0.85 & & $\mathrm{~d}$ & \\
\hline 56108 & 5.8 & 1.15 & 0.73 & & & 23.16 & 345 & & 2.20 & 4225 & 33.96 & -20.34 & -0.06 & -0.53 & & $\mathrm{~d}$ & \\
\hline 56334 & 1.9 & 1.44 & 0.14 & -0.04 & 0.59 & 23.62 & 178 & & 1.99 & 1853 & 32.28 & -19.77 & 0.13 & -0.46 & & $\mathrm{~d}$ & \\
\hline 56925 & 3.2 & 1.00 & 0.21 & & & 22.30 & & & & 3370 & 33.51 & -20.13 & & & & & \\
\hline 57471 & 2.0 & 0.96 & 0.28 & & & 23.57 & & & & 3904 & 33.88 & -18.90 & -1.15 & & & $\mathrm{~d}$ & \\
\hline 58115 & 5.7 & 1.13 & 0.18 & & & 22.90 & 201 & & 2.05 & 2416 & 32.92 & -19.44 & -1.28 & -0.87 & & $\mathrm{~d}$ & \\
\hline 58183 & -2.0 & 1.08 & 0.27 & & & 23.78 & & & & 2409 & 32.84 & -18.50 & & & & & \\
\hline 58336 & 5.8 & 1.22 & 0.45 & & & 24.85 & 243 & & 2.05 & 2988 & 33.28 & -18.40 & & 0.31 & & $\mathrm{~d}$ & \\
\hline 58633 & 4.5 & 1.18 & 0.65 & & & 24.34 & 123 & & 1.68 & 851 & 30.96 & -16.20 & & -0.03 & & $\mathrm{~d}$ & \\
\hline 58827 & 4.9 & 1.47 & 0.38 & -0.25 & 0.44 & 22.47 & 240 & & 2.05 & 851 & 30.96 & -19.59 & -0.03 & -0.76 & -1.47 & $\mathrm{~b}, \mathrm{~d}$ & \\
\hline 69898 & 5.9 & 1.27 & 0.05 & & & 24.73 & 204 & & 2.27 & 3504 & 33.52 & -19.27 & & -0.22 & & $\mathrm{~d}$ & \\
\hline 71133 & 1.2 & 1.18 & 0.01 & 0.13 & 0.70 & 22.50 & 207 & & 2.59 & 1623 & 31.93 & -19.22 & -2.03 & -0.44 & -0.50 & $\mathrm{~b}, \mathrm{c}, \mathrm{d}$ & ARP212, VV280 \\
\hline 71360 & 1.0 & 1.03 & 0.60 & & & 23.69 & & & & 3033 & 33.21 & -18.59 & & & & d & \\
\hline 71699 & 4.0 & 1.28 & 0.10 & & & 23.95 & 228 & & 2.20 & 4042 & 33.84 & -20.31 & -0.06 & -0.47 & & d & \\
\hline 71796 & 4.0 & 0.87 & 0.19 & & & 23.20 & 201 & & 2.02 & 4168 & 33.91 & -19.01 & & -0.22 & & $\mathrm{~d}$ & \\
\hline 73163 & 4.2 & 1.14 & 0.04 & -0.21 & 0.51 & 22.34 & 96 & & 1.94 & 2404 & 32.75 & -20.15 & -1.27 & -1.15 & & $\mathrm{~d}$ & \\
\hline
\end{tabular}

Notes: the presence of the galaxy in Arp or Vorontsov Velyaminov atlases is reported with the original name (ARP and VV). The presence of peculiar kinematics such as counterrotation is indicated with CR. REFERENCES: a: Boselli, A., Gavazzi, G., Lequeux, J., Buat, V., Casoli, F., Dickey, J., \& Donas, J. 1995, A\&A, 300, L13; b: Young, J. S., Xie Shuding, Tacconi, L. J., et al. 1995, ApJS, 98, 219; c: Knapp, G. R., Guhathakurta, P., Kim, D.-W., \& Jura, M. 1989, ApJS, 70, 329; d: (LEDA) Paturel, G., Andernach, H., Bottinelli, L., Di Nella, H., Durand, N., Garnier, R., Gouguenheim, L., Lanoix, P., Martinet, M. C., Petit, C., Rousseau, J., Theureau, G., \& Vauglin, I. 1997, A\&AS, 124, 109; e: Roberts, M., Hogg, D. E., Bregman, J. N., Forman, W. R., \& Jones, C. 1991, ApJS, 75, 751; f: Sage, L. J. 1993, A\&A, 100, 537 and A\&A, 272, 123; g: van Driel, W., Ragaigne, D., Boselli, A., Donas, J., \& Gavazzi, G. 2000, A\&AS, 144, 463; h: Zhu, Ming, Seaquist, E. R., Davoust, Emmanuel, Frayer, David, T., Bushouse, Howard 1999, AJ, 118, 145. 
Table 2. Main properties of the Perturbed galaxies. All the propertis were extracted form LEDA except the molecular gas content

\begin{tabular}{|c|c|c|c|c|c|c|c|c|c|c|c|c|c|c|c|c|c|}
\hline PGC & $t$ & $\begin{array}{r}\log D_{25} \\
\left(0^{\prime} \cdot 1\right)\end{array}$ & $\begin{array}{r}\log r_{25} \\
(0.1)\end{array}$ & $(U-B)_{0}$ & $(B-V)_{0}$ & $\begin{array}{c}\mu_{25} \\
\left(\mathrm{ma} / \operatorname{arcsec}^{2}\right)\end{array}$ & $\begin{array}{r}W_{20} \\
\left(\mathrm{~km} \mathrm{~s}^{-1}\right)\end{array}$ & $\begin{array}{r}\log \sigma \\
\left(\mathrm{km} \mathrm{s}^{-1}\right)\end{array}$ & $\begin{array}{r}\log v_{\mathrm{m}} \\
\left(\mathrm{km} \mathrm{s}^{-1}\right)\end{array}$ & $\begin{array}{c}c z \\
\left(\mathrm{~km} \mathrm{~s}^{-1}\right)\end{array}$ & $(m-M)_{\operatorname{cin}}$ & $M_{\mathrm{B}}$ & FIR-B & $\log \frac{M_{\mathrm{HI}}}{L_{\mathrm{B}}}$ & $\log \frac{M_{\mathrm{mol}}}{L_{\mathrm{B}}}$ & Ref. & Notes \\
\hline 696 & -0.1 & 1.08 & 0.12 & & & 24.31 & & & & 4031 & 33.80 & -18.79 & & & & & \\
\hline 2357 & -1.9 & 1.37 & 0.42 & 0.36 & 0.84 & 24.56 & & & & 4231 & 33.89 & -20.02 & & & & & \\
\hline 2365 & -1.2 & 0.97 & 0.10 & 0.05 & 0.59 & 23.52 & & 2.30 & & 4154 & 33.85 & -19.03 & & & & & \\
\hline 4777 & 3.1 & 1.45 & 0.26 & 0.02 & 0.65 & 23.12 & 394.08 & & 2.32 & 2374 & 32.63 & -20.63 & -0.89 & -0.78 & $<-0.96$ & $\mathrm{~b}, \mathrm{~d}$ & \\
\hline 4801 & -2.0 & 1.79 & 0.09 & 0.35 & 0.80 & 25.03 & 367.85 & 2.23 & 2.41 & 2365 & 32.62 & -20.44 & & -1.32 & & $\mathrm{c}, \mathrm{d}, \mathrm{e}$ & ARP227 \\
\hline 7525 & 3.0 & 1.85 & 0.25 & 0.15 & 0.65 & 23.80 & 472.06 & 2.15 & 2.47 & 2456 & 32.76 & -22.23 & 0.38 & -0.66 & -0.60 & $\mathrm{~b}, \mathrm{~d}$ & ARP78 \\
\hline 7533 & 6.5 & 1.15 & 0.33 & & & 24.87 & 190.50 & & 1.96 & 4613 & 34.10 & -18.92 & & -0.34 & & $\mathrm{~d}$ & \\
\hline 7846 & -1.9 & 1.18 & 0.24 & 0.41 & 0.85 & 23.72 & & & & 3452 & 33.47 & -19.66 & & & & & ARP290, VV309 \\
\hline 7856 & 3.1 & 1.40 & 0.42 & & & 23.97 & 381.50 & 2.10 & 2.26 & 3646 & 33.59 & -20.53 & & -0.82 & & $\mathrm{~d}$ & ARP290, VV309 \\
\hline 8360 & 6.0 & 1.34 & 0.20 & & & 24.73 & 231.57 & & 2.09 & 3256 & 33.31 & -19.17 & & 0.09 & & $\mathrm{~d}$ & \\
\hline 26232 & 1.1 & 1.43 & 0.46 & -0.09 & 0.60 & 23.45 & 316.11 & & 2.12 & 1739 & 32.17 & -19.62 & -2.66 & -0.82 & -0.42 & $\mathrm{~b}, \mathrm{~d}, \mathrm{e}$ & ARP283, VV50 \\
\hline 26498 & 5.4 & 1.65 & 1.04 & & 0.23 & 23.41 & 370.13 & & 2.22 & 1580 & 32.07 & -20.70 & 0.21 & -0.18 & & $\mathrm{~d}$ & \\
\hline 26571 & 1.0 & 1.17 & 0.03 & & & 23.67 & 230.35 & 2.28 & 2.37 & 1782 & 32.21 & -18.19 & & -0.47 & & $\mathrm{~d}$ & \\
\hline 26580 & -0.9 & 1.27 & 0.25 & & & 24.45 & & & & 1765 & 32.19 & -17.88 & & & & & \\
\hline 27159 & -2.1 & 1.57 & 0.17 & 0.43 & 0.89 & 24.27 & 518.66 & 2.37 & 2.40 & 3179 & 33.30 & -20.79 & & -1.17 & & $\mathrm{c}, \mathrm{d}, \mathrm{e}$ & ARP232 \\
\hline 27939 & 1.6 & 1.16 & 0.06 & & & 23.27 & & & 2.39 & 4430 & 34.13 & -20.87 & 0.09 & -1.29 & & $\mathrm{~d}$ & \\
\hline 28197 & 5.8 & 1.21 & 0.83 & & & 23.56 & & & 2.04 & 3025 & 33.35 & -19.68 & & -0.25 & & $\mathrm{~d}$ & \\
\hline 29814 & 0.2 & 1.64 & 0.29 & 0.32 & 0.84 & 23.50 & 139.08 & 2.11 & 1.82 & 1331 & 31.39 & -19.92 & -0.34 & -1.59 & -1.29 & $\mathrm{c}, \mathrm{d}, \mathrm{e}$ & \\
\hline 29855 & 1.2 & 1.67 & 0.27 & 0.19 & 0.76 & 22.90 & 508.46 & 2.23 & 2.25 & 1233 & 31.22 & -20.50 & 0.09 & -0.63 & -0.61 & $b, d$ & \\
\hline 30068 & 5.0 & 1.38 & 0.41 & -0.28 & 0.36 & 23.85 & 262.37 & & 2.07 & 1578 & 31.88 & -18.72 & -0.16 & -0.52 & & $\mathrm{~d}$ & ARP316, VV307 \\
\hline 30083 & 0.9 & 1.63 & 0.41 & 0.38 & 0.86 & 23.58 & 527.87 & 2.24 & 2.39 & 1312 & 31.51 & -19.87 & -0.10 & -1.48 & & $\mathrm{c}, \mathrm{d}, \mathrm{e}$ & ARP316, VV307 \\
\hline 30445 & 1.4 & 1.70 & 0.23 & 0.21 & 0.75 & 23.32 & 418.71 & 2.13 & 2.35 & 1154 & 31.23 & -20.19 & -0.25 & -1.09 & & $\mathrm{~d}$ & ARP94, VV209 \\
\hline 30714 & 3.4 & 1.50 & 0.99 & & & 25.13 & 198.50 & & 1.90 & 1324 & 31.58 & -17.59 & & 0.07 & & $\mathrm{~d}$ & \\
\hline 32292 & -2.6 & 1.73 & 0.30 & 0.40 & 0.88 & 23.15 & 84.75 & 2.15 & 1.38 & 890 & 30.65 & -19.97 & & -2.15 & & $\mathrm{c}, \mathrm{d}, \mathrm{e}, \mathrm{g}$ & \\
\hline 32306 & 5.3 & 1.45 & 0.35 & -0.23 & 0.36 & 22.70 & 275.17 & & 2.15 & 1291 & 31.42 & -19.78 & & -0.79 & & $\mathrm{~d}$ & \\
\hline 32533 & -2.1 & 1.50 & 0.14 & 0.52 & 0.92 & 23.32 & 324.42 & 2.39 & 2.27 & 1524 & 31.86 & -19.92 & & -1.86 & & $\mathrm{c}, \mathrm{d}, \mathrm{e}, \mathrm{g}$ & ARP162 \\
\hline 32584 & 3.1 & 1.44 & 0.55 & & & 23.14 & 399.35 & & 2.24 & 1503 & 31.86 & -19.66 & -1.48 & -0.69 & & $\mathrm{~d}$ & \\
\hline 32605 & 5.2 & 1.52 & 0.03 & & & 24.41 & 271.48 & & 2.54 & 2719 & 32.98 & -20.07 & & -0.21 & & $\mathrm{~d}$ & \\
\hline 32767 & 3.2 & 1.42 & 0.27 & & & 23.95 & 215.36 & & 2.02 & 1107 & 31.14 & -18.11 & & -0.28 & & $\mathrm{~d}$ & \\
\hline 34029 & 6.0 & 1.09 & 0.06 & & & 24.58 & 124.23 & & 1.97 & 3052 & 33.36 & -18.01 & & 0.24 & & $\mathrm{~d}$ & \\
\hline
\end{tabular}


Table 2. continued.

\begin{tabular}{|c|c|c|c|c|c|c|c|c|c|c|c|c|c|c|c|c|c|}
\hline PGC & $t$ & $\begin{array}{r}\log D_{25} \\
(0.1)\end{array}$ & $\begin{array}{r}\log r_{25} \\
\left(0^{\prime} 1\right)\end{array}$ & $(U-B)_{0}$ & $(B-V)_{0}$ & $\begin{array}{c}\mu_{25} \\
\left(\mathrm{ma} / \operatorname{arcsec}^{2}\right)\end{array}$ & $\begin{array}{r}W_{20} \\
\left(\mathrm{~km} \mathrm{~s}^{-1}\right)\end{array}$ & $\begin{array}{r}\log \sigma \\
\left(\mathrm{km} \mathrm{s}^{-1}\right)\end{array}$ & $\begin{array}{r}\log v_{\mathrm{m}} \\
\left(\mathrm{km} \mathrm{s}^{-1}\right)\end{array}$ & $\begin{array}{c}c z \\
\left(\mathrm{~km} \mathrm{~s}^{-1}\right)\end{array}$ & $(m-M)_{\mathrm{cin}}$ & $M_{\mathrm{B}}$ & FIR-B & $\log \frac{M_{\mathrm{HI}}}{L_{\mathrm{B}}}$ & $\log \frac{M_{\mathrm{mol}}}{L_{\mathrm{B}}}$ & Ref. & Notes \\
\hline 34415 & -4.6 & 0.97 & 0.20 & 0.44 & 0.83 & 21.89 & & 1.99 & & 661 & 30.15 & -17.07 & & $<-2.03$ & & $\mathrm{e}$ & \\
\hline 34561 & 5.3 & 1.62 & 0.18 & & & 24.42 & 300.53 & & 2.24 & 2331 & 32.80 & -20.23 & 0.36 & -0.25 & & $\mathrm{~d}$ & \\
\hline 35616 & 1.1 & 1.88 & 0.27 & 0.23 & 0.74 & 24.39 & 474.00 & 2.25 & 2.40 & 992 & 31.21 & -19.97 & 1.88 & -0.33 & $<-1.22$ & $\mathrm{~b}, \mathrm{c}, \mathrm{d}$ & ARP214 \\
\hline 35999 & 3.4 & 1.44 & 0.50 & & & 22.73 & 255.24 & & 2.06 & 730 & 30.66 & -18.89 & & -0.40 & & $\mathrm{~d}$ & ARP280 \\
\hline 36060 & 6.4 & 1.11 & 0.33 & -0.18 & 0.39 & 23.72 & 222.94 & & 2.03 & 3358 & 33.48 & -19.09 & & -0.27 & & $\mathrm{~d}$ & \\
\hline 36158 & 1.1 & 1.30 & 0.28 & & & 23.66 & 450.89 & 2.15 & 2.35 & 2718 & 33.07 & -19.74 & & -0.36 & & $\mathrm{~d}$ & ARP294, VV228 \\
\hline 36160 & 2.2 & 1.23 & 0.57 & & & 22.76 & 541.72 & & 2.42 & 2689 & 33.05 & -20.21 & & -0.57 & & $\mathrm{~d}$ & ARP294, VV228 \\
\hline 36193 & 3.1 & 0.85 & 0.21 & & & 21.94 & 438.28 & & 2.40 & 3312 & 33.43 & -19.62 & & -0.71 & & $\mathrm{~d}$ & ARP83, VV350 \\
\hline 36197 & 3.1 & 1.24 & 0.52 & & & 22.89 & 430.32 & & 2.31 & 3306 & 33.43 & -20.56 & -1.59 & -0.97 & & $\mathrm{~d}$ & ARP83, VV350 \\
\hline 36200 & -1.8 & 1.46 & 0.22 & 0.35 & 0.84 & 23.99 & 329.75 & 2.25 & 2.21 & 3319 & 33.44 & -20.65 & & -0.98 & & $\mathrm{c}, \mathrm{d}$ & \\
\hline 36871 & 4.7 & 0.78 & 0.51 & & & 22.63 & & & & 4394 & 34.03 & -19.12 & & & & & \\
\hline 36897 & 0.2 & 1.18 & 0.16 & & 0.49 & 23.58 & 277.90 & & 2.18 & 955 & 31.13 & -17.25 & & 0.42 & & $\mathrm{~d}$ & \\
\hline 36907 & 1.1 & 1.12 & 0.17 & 0.26 & 0.86 & 23.14 & & & & 3301 & 33.53 & -19.89 & & & & 3 & \\
\hline 37466 & 4.0 & 1.57 & 0.57 & -0.14 & 0.54 & 23.61 & 267.13 & & 2.07 & 845 & 30.96 & -18.90 & 0.65 & -0.80 & & $\mathrm{~d}$ & \\
\hline 37618 & -2.7 & 1.13 & 0.19 & 0.36 & 0.85 & 22.85 & & 2.25 & & 695 & 30.64 & -17.26 & & & & $\mathrm{c}$ & \\
\hline 37619 & 3.1 & 1.21 & 0.54 & & & 23.22 & 374.18 & & 2.24 & 4826 & 34.25 & -20.86 & & -0.74 & 0.07 & $b, d$ & \\
\hline 37629 & 3.0 & 1.16 & 0.28 & -0.15 & 0.47 & 23.35 & 280.67 & & 2.13 & 4768 & 34.23 & -20.49 & -0.82 & -0.39 & -1.59 & $\mathrm{a}, \mathrm{d}$ & \\
\hline 37642 & -2.1 & 1.46 & 0.08 & 0.50 & 0.92 & 22.59 & 633.00 & 2.48 & 2.67 & 1042 & 31.32 & -19.83 & & -1.41 & & $\mathrm{c}, \mathrm{d}, \mathrm{e}$ & \\
\hline 37692 & 5.9 & 1.35 & 0.09 & -0.07 & 0.52 & 23.29 & 215.83 & & 2.15 & 767 & 30.72 & -18.00 & & -0.38 & & $\mathrm{~d}$ & \\
\hline 37719 & 2.2 & 1.41 & 0.37 & 0.26 & 0.91 & 23.21 & 358.40 & 2.12 & 2.24 & 699 & 30.56 & -18.15 & & -0.54 & & $\mathrm{~d}$ & \\
\hline 38287 & 1.3 & 1.02 & 0.50 & & & 22.73 & 411.05 & 2.24 & 2.28 & 4275 & 33.98 & -20.18 & & -0.48 & & $\mathrm{~d}$ & \\
\hline 38503 & -2.0 & 1.29 & 0.41 & & & 24.28 & 283.50 & 1.98 & 2.09 & 933 & 31.06 & -16.97 & & -0.01 & & $\mathrm{~d}$ & \\
\hline 38885 & 1.9 & 1.08 & 0.22 & 0.14 & 0.75 & 23.28 & 270.24 & & 2.14 & 1862 & 32.22 & -18.23 & & -1.24 & & $\mathrm{~d}$ & \\
\hline 38892 & -1.9 & 1.12 & 0.27 & 0.42 & 0.87 & 22.62 & 476.10 & 2.34 & 2.36 & 3814 & 33.77 & -20.60 & & -0.86 & & $\mathrm{c}, \mathrm{d}$ & \\
\hline 38906 & -0.2 & 0.84 & 0.35 & & & 22.31 & 423.57 & & 2.19 & 3945 & 33.84 & -19.54 & & -0.04 & & $\mathrm{~d}, \mathrm{~g}$ & \\
\hline 38912 & 3.2 & 1.14 & 0.63 & & & 22.74 & 261.72 & & 2.03 & 3941 & 33.84 & -20.53 & -2.06 & -0.60 & -0.58 & $\mathrm{a}, \mathrm{d}$ & \\
\hline 39568 & 3.0 & 1.61 & 0.78 & & & 23.52 & 545.50 & & 2.42 & 2529 & 33.01 & -21.28 & 1.73 & -1.23 & & $\mathrm{~d}$ & \\
\hline 39687 & -1.0 & 0.95 & 0.11 & 0.44 & 0.82 & 22.22 & & & & 2632 & 32.92 & -19.30 & & & & $\mathrm{c}$ & \\
\hline 39708 & -1.9 & 0.99 & 0.34 & & & 23.71 & 393.90 & & 2.26 & 2291 & 32.63 & -17.66 & & -0.12 & & $\mathrm{~d}$ & \\
\hline 39712 & -0.2 & 1.17 & 0.37 & 0.41 & 0.94 & 23.09 & 292.41 & & 2.07 & 2374 & 32.70 & -19.23 & & -0.51 & & $\mathrm{c}, \mathrm{d}$ & \\
\hline 39719 & -1.0 & 1.12 & 0.14 & & & 23.16 & & & & 2208 & 32.55 & -18.83 & & & & $\mathrm{c}$ & \\
\hline 39759 & 0.2 & 0.91 & 0.27 & & 0.92 & 23.11 & 278.75 & & 2.13 & 2504 & 32.81 & -18.04 & & -0.03 & & $\mathrm{~d}$ & \\
\hline
\end{tabular}


Table 2. continued.

\begin{tabular}{|c|c|c|c|c|c|c|c|c|c|c|c|c|c|c|c|c|c|}
\hline PGC & $t$ & $\begin{array}{r}\log D_{25} \\
(0.1)\end{array}$ & $\begin{array}{r}\log r_{25} \\
\left(0^{\prime} 1\right)\end{array}$ & $(U-B)_{0}$ & $(B-V)_{0}$ & $\begin{array}{c}\mu_{25} \\
\left(\mathrm{ma} / \operatorname{arcsec}^{2}\right)\end{array}$ & $\begin{array}{r}W_{20} \\
\left(\mathrm{~km} \mathrm{~s}^{-1}\right)\end{array}$ & $\begin{array}{r}\log \sigma \\
\left(\mathrm{km} \mathrm{s}^{-1}\right)\end{array}$ & $\begin{array}{r}\log v_{\mathrm{m}} \\
\left(\mathrm{km} \mathrm{s}^{-1}\right)\end{array}$ & $\begin{array}{c}c z \\
\left(\mathrm{~km} \mathrm{~s}^{-1}\right)\end{array}$ & $(m-M)_{\operatorname{cin}}$ & $M_{\mathrm{B}}$ & FIR-B & $\log \frac{M_{\mathrm{HI}}}{L_{\mathrm{B}}}$ & $\log \frac{M_{\mathrm{mol}}}{L_{\mathrm{B}}}$ & Ref. & Notes \\
\hline 39943 & -2.0 & 1.03 & 0.27 & & 0.73 & 23.10 & & 2.29 & & 4229 & 33.94 & -19.85 & & & & $\mathrm{c}$ & \\
\hline 39950 & 5.2 & 1.47 & 0.26 & & 0.61 & 22.64 & 264.75 & & 2.11 & 1140 & 31.24 & -19.78 & -0.42 & -1.11 & -0.62 & $\mathrm{~b}, \mathrm{~d}$ & \\
\hline 39974 & 5.4 & 1.69 & 0.82 & & 0.72 & 23.25 & 376.58 & & 2.24 & 1118 & 31.20 & -20.15 & & -0.60 & -0.81 & $\mathrm{~b}, \mathrm{~d}$ & \\
\hline 39981 & 2.4 & 1.43 & 0.28 & & & 23.57 & & & & 1484 & 32.01 & -19.45 & & & & d & \\
\hline 40030 & 1.0 & 1.30 & 0.26 & & 0.59 & 23.30 & & & & 1893 & 32.26 & -19.34 & & & & & \\
\hline 40032 & -2.0 & 1.16 & 0.16 & & & 23.53 & & & & 1650 & 31.98 & -18.16 & & & & & \\
\hline 40245 & -1.2 & 1.51 & 0.11 & 0.46 & 0.88 & 23.36 & & 2.07 & & 917 & 30.83 & -18.85 & & $<-2.23$ & & $\mathrm{c}, \mathrm{e}$ & \\
\hline 40295 & -1.8 & 1.46 & 0.28 & 0.42 & 0.88 & 22.91 & & 2.29 & & 1226 & 31.40 & -19.63 & & $<-2.26$ & & $\mathrm{c}, \mathrm{e}$ & \\
\hline 40309 & -1.8 & 1.06 & 0.06 & & & 23.76 & & & & 4496 & 34.16 & -19.57 & & & & & \\
\hline 40515 & -1.3 & 1.85 & 0.11 & 0.38 & 0.84 & 23.02 & & 2.26 & & 742 & 30.44 & -20.55 & & $<-3.13$ & & $\mathrm{c}, \mathrm{e}$ & \\
\hline 40581 & 2.8 & 1.74 & 0.59 & 0.15 & 0.58 & 23.41 & 386.07 & 1.93 & 2.33 & 2519 & 32.86 & -21.92 & -0.34 & -1.53 & -1.28 & $a, b, d$ & \\
\hline 40614 & 2.9 & 1.54 & 0.02 & 0.18 & 0.81 & 23.12 & 177.64 & 2.15 & 2.32 & 921 & 30.85 & -19.30 & 1.09 & -1.35 & -0.95 & $\mathrm{~b}, \mathrm{~d}$ & \\
\hline 40836 & -1.0 & 1.59 & 0.11 & 0.26 & 0.61 & 25.04 & & 2.20 & & 2662 & 33.11 & -19.87 & -1.06 & & & $\mathrm{c}, \mathrm{d}$ & \\
\hline 40903 & -1.8 & 1.17 & 0.27 & 0.41 & 0.86 & 23.48 & & & & 1211 & 31.34 & -17.55 & & & & & \\
\hline 41302 & -1.8 & 1.18 & 0.06 & 0.47 & 0.92 & 23.15 & & 1.91 & & 850 & 30.65 & -17.29 & & & & $\mathrm{c}$ & \\
\hline 41363 & 0.1 & 1.56 & 0.50 & 0.30 & 0.76 & 24.43 & & & & 994 & 30.91 & -18.05 & & & & $\mathrm{c}$ & \\
\hline 42064 & 4.0 & 1.44 & 0.13 & 0.26 & 0.68 & 22.82 & 320.95 & & 2.25 & 2265 & 32.63 & -20.88 & & -0.78 & -0.62 & $b, d$ & VV219 \\
\hline 42069 & 4.1 & 1.64 & 0.34 & & 0.76 & 22.98 & & & 2.27 & 2255 & 32.62 & -21.66 & -1.07 & -1.39 & -0.61 & $b, d$ & VV219 \\
\hline 42620 & -4.3 & 1.32 & 0.12 & 0.09 & 0.61 & 23.52 & & & & 765 & 30.65 & -17.69 & & & & 1 & ARP281 \\
\hline 42710 & 8.5 & 0.71 & 0.17 & & & 23.30 & 160.75 & & 1.90 & 1100 & 31.17 & -15.22 & & 0.14 & & $\mathrm{~d}$ & \\
\hline 42728 & -2.7 & 1.39 & 0.16 & 0.40 & 0.89 & 22.80 & & 2.11 & & 1127 & 31.20 & -19.22 & & $<-2.12$ & & $\mathrm{e}$ & \\
\hline 42816 & 5.2 & 1.45 & 0.08 & 0.25 & 0.60 & 22.76 & 212.45 & & 2.09 & 1415 & 31.66 & -19.99 & -0.57 & -1.22 & -0.57 & $\mathrm{~b}, \mathrm{~d}$ & ARP116, VV206 \\
\hline 47777 & 4.9 & 1.05 & 0.62 & & & 23.29 & & & & 4600 & 34.15 & -19.85 & -1.66 & & & $\mathrm{~d}$ & \\
\hline 47867 & 3.3 & 1.05 & 0.10 & & 0.37 & 24.24 & 226.84 & & 2.17 & 4962 & 34.34 & -19.16 & & 0.31 & & $\mathrm{~d}$ & ARP183 \\
\hline 48018 & & 0.84 & 0.34 & & & 24.19 & & & & 3886 & 33.75 & -17.61 & & & & & \\
\hline 48811 & -1.1 & 0.98 & 0.22 & 0.35 & 0.56 & 23.88 & & 2.43 & & 7333 & 35.17 & -20.05 & & & & & \\
\hline 48815 & 4.9 & 1.71 & 0.63 & -0.06 & 0.49 & 23.60 & 415.44 & 2.21 & 2.29 & 2403 & 32.88 & -21.54 & 0.58 & -0.50 & & $\mathrm{~d}$ & $\mathrm{CR}$ \\
\hline 48860 & -2.0 & 1.56 & 0.74 & & 0.82 & 23.74 & & 2.42 & 2.18 & 2021 & 32.58 & -20.38 & & -1.58 & & $\mathrm{c}, \mathrm{d}, \mathrm{e}$ & \\
\hline 49347 & 3.6 & 1.43 & 0.14 & 0.24 & 0.76 & 23.00 & 309.40 & & 2.28 & 2315 & 32.80 & -20.74 & -0.21 & -0.53 & & $\mathrm{~d}$ & \\
\hline 49354 & -2.1 & 1.32 & 0.18 & 0.49 & 0.91 & 22.83 & 288.90 & & 2.16 & 2305 & 32.79 & -20.38 & & -0.56 & & $\mathrm{c}, \mathrm{d}$ & CR \\
\hline 49356 & -2.1 & 1.30 & 0.20 & 0.58 & 0.92 & 22.38 & 295.90 & 2.45 & 2.18 & 2305 & 32.79 & -20.73 & & -0.74 & & $\mathrm{c}, \mathrm{d}, \mathrm{e}$ & \\
\hline 49508 & -1.8 & 1.35 & 0.36 & & & 24.56 & 158.50 & & 1.80 & 1762 & 32.32 & -18.27 & & -0.04 & & $\mathrm{~d}$ & \\
\hline
\end{tabular}


Table 2. continued.

\begin{tabular}{|c|c|c|c|c|c|c|c|c|c|c|c|c|c|c|c|c|c|}
\hline PGC & $t$ & $\begin{array}{r}\log D_{25} \\
\left(0^{\prime} 1\right)\end{array}$ & $\begin{array}{r}\log r_{25} \\
\left(0^{\prime} 1\right)\end{array}$ & $(U-B)_{0}$ & $(B-V)_{0}$ & $\begin{array}{c}\mu_{25} \\
\left(\mathrm{ma} / \operatorname{arcsec}^{2}\right)\end{array}$ & $\begin{array}{r}W_{20} \\
\left(\mathrm{~km} \mathrm{~s}^{-1}\right)\end{array}$ & $\begin{array}{r}\log \sigma \\
\left(\mathrm{km} \mathrm{s}^{-1}\right)\end{array}$ & $\begin{array}{r}\log v_{\mathrm{m}} \\
\left(\mathrm{km} \mathrm{s}^{-1}\right)\end{array}$ & $\begin{array}{c}c z \\
\left(\mathrm{~km} \mathrm{~s}^{-1}\right)\end{array}$ & $(m-M)_{\mathrm{cin}}$ & $M_{\mathrm{B}}$ & FIR-B & $\log \frac{M_{\mathrm{HI}}}{L_{\mathrm{B}}}$ & $\log \frac{M_{\text {mol }}}{L_{\mathrm{B}}}$ & Ref. & Notes \\
\hline 49548 & -0.4 & 1.60 & 0.56 & 0.34 & 0.78 & 24.44 & & 2.22 & & 1842 & 32.40 & -19.68 & 1.15 & & & $\mathrm{c}, \mathrm{d}$ & \\
\hline 49739 & 3.1 & 1.28 & 0.26 & 0.06 & 0.54 & 23.46 & 595.50 & & 2.50 & 3472 & 33.61 & -20.30 & -2.29 & -0.22 & -0.39 & $\mathrm{~d}, \mathrm{a}$ & ARP84, VV48 \\
\hline 49747 & 3.2 & 1.41 & 0.28 & 0.04 & 0.53 & 22.42 & 625.93 & & 2.51 & 3486 & 33.62 & -21.98 & & -0.70 & -0.62 & $\mathrm{~d}, \mathrm{a}$ & ARP84, VV48 \\
\hline 49820 & 3.0 & 1.47 & 0.57 & & & 23.84 & 512.13 & & 2.39 & 2743 & 33.13 & -20.35 & -1.18 & -0.23 & & d & VV310 \\
\hline 49824 & 0.1 & 0.63 & 0.28 & & & 22.11 & & & & 2748 & 33.14 & -17.93 & & & & & VV310 \\
\hline 49893 & 4.9 & 1.13 & 0.28 & & & 23.85 & 262.85 & & 2.11 & 3721 & 33.76 & -19.34 & -1.15 & -0.09 & -0.62 & $\mathrm{~d}, \mathrm{a}$ & VV256 \\
\hline 50331 & -4.0 & 1.26 & 0.13 & & & 23.81 & & 2.17 & & 1885 & 32.43 & -18.90 & & & & $\mathrm{c}$ & \\
\hline 50776 & -2.0 & 1.28 & 0.23 & & & 24.02 & & 2.14 & & 4991 & 34.33 & -20.64 & & & & & \\
\hline 51223 & 3.0 & 1.56 & 0.61 & 0.04 & 0.68 & 23.76 & 254.06 & & 2.02 & 1728 & 32.06 & -19.84 & 0.03 & -1.06 & & d & ARP286 \\
\hline 51233 & 1.5 & 1.82 & 0.46 & 0.34 & 0.78 & 23.69 & 504.51 & & 2.43 & 1506 & 31.78 & -20.99 & 1.65 & -1.36 & & $\mathrm{~d}, \mathrm{e}$ & ARP286 \\
\hline 51241 & 5.8 & 1.15 & 0.09 & & & 24.75 & 105.53 & & 1.83 & 1773 & 32.12 & -16.98 & & 0.14 & & d & ARP286 \\
\hline 51270 & -2.7 & 1.16 & 0.17 & 0.28 & 0.78 & 23.04 & 613.60 & & 2.38 & 1656 & 31.97 & -18.63 & & -1.55 & & $\mathrm{c}, \mathrm{d}, \mathrm{e}$ & \\
\hline 51668 & -1.9 & 1.01 & 0.19 & & & 23.57 & 183.00 & 2.24 & 1.93 & 4370 & 34.07 & -19.42 & & -1.17 & & $\mathrm{~d}$ & \\
\hline 51681 & -2.0 & 1.24 & 0.02 & & 0.90 & 23.13 & & 2.43 & & 4518 & 34.14 & -21.14 & & & & $\mathrm{c}$ & \\
\hline 51785 & -0.4 & 1.15 & 0.17 & & & 23.86 & & & 2.31 & 1676 & 32.00 & -17.74 & & -1.02 & & d & \\
\hline 51965 & 3.2 & 1.42 & 0.48 & & & 23.69 & & & & 4068 & 33.94 & -21.08 & 0.52 & & & d & \\
\hline 52686 & 3.0 & 1.22 & 0.06 & & & 23.57 & 245.90 & 2.24 & 2.31 & 4400 & 34.10 & -20.42 & & -0.58 & & d & ARP297 \\
\hline 53176 & 4.5 & 1.08 & 0.26 & & & 23.24 & 219.05 & 2.03 & 2.03 & 1576 & 31.88 & -17.96 & & -0.36 & & d & \\
\hline 53178 & 3.0 & 1.30 & 0.09 & & & 23.36 & 223.72 & & 2.20 & 1577 & 31.88 & -18.97 & & -0.72 & & $\mathrm{~d}$ & \\
\hline 53217 & 5.2 & 1.46 & 0.21 & & & 24.60 & 263.17 & & 2.15 & 2334 & 32.85 & -19.43 & 0.38 & -0.17 & & d & \\
\hline 53657 & 4.0 & 1.24 & 0.68 & & & 23.34 & 312.85 & & 2.13 & 2544 & 33.01 & -19.58 & -1.09 & 0.16 & & d & \\
\hline 53995 & 2.5 & 1.08 & 0.29 & -0.13 & 0.64 & 22.52 & & 2.22 & 2.38 & 4759 & 34.24 & -20.98 & & & & & \\
\hline 54001 & 4.0 & 1.44 & 0.55 & 0.07 & 0.65 & 23.27 & 479.70 & & 2.37 & 4756 & 34.24 & -21.98 & -0.29 & -0.75 & & d & \\
\hline 55647 & 4.3 & 1.43 & 0.89 & 0.23 & 0.76 & 23.30 & & & 2.42 & 2527 & 33.02 & -20.54 & 0.75 & -0.45 & & d & \\
\hline 55725 & 3.1 & 1.71 & 0.30 & 0.08 & 0.68 & 23.73 & 534.80 & & 2.49 & 2519 & 33.01 & -21.61 & 0.99 & -0.79 & & d & \\
\hline 57579 & -3.3 & 0.92 & 0.06 & & & 23.26 & & & & 9444 & 35.72 & -21.04 & & & & & \\
\hline 57627 & 5.1 & 1.34 & 0.80 & & & 23.67 & 226.50 & & 1.99 & 2306 & 32.74 & -19.59 & & -0.63 & & d & \\
\hline 69630 & 0.3 & 1.22 & 0.18 & & & 23.78 & 394.22 & & 2.35 & 4851 & 34.21 & -20.59 & & -0.42 & & d & \\
\hline 70348 & 1.1 & 1.16 & 0.12 & -0.46 & 0.48 & 22.38 & 386.28 & & 2.41 & 4916 & 34.25 & -21.77 & -2.79 & -1.35 & -0.64 & $\mathrm{~b}, \mathrm{~d}$ & ARP298 \\
\hline 70786 & 3.6 & 1.31 & 0.59 & -0.19 & 0.46 & 23.14 & 363.39 & 2.07 & 2.16 & 2676 & 32.93 & -20.29 & & -0.28 & & $\mathrm{~d}$ & \\
\hline 70795 & 4.7 & 1.52 & 0.46 & -0.06 & 0.53 & 22.90 & 469.93 & 1.83 & 2.33 & 2683 & 32.93 & -21.60 & -1.44 & -0.57 & -0.67 & $\mathrm{~b}, \mathrm{~d}$ & \\
\hline 71034 & 6.8 & 1.16 & 0.50 & -0.24 & 0.37 & 23.97 & 227.62 & & 1.97 & 4198 & 33.91 & -19.84 & & -0.50 & & d & \\
\hline 71113 & -1.9 & 0.97 & 0.14 & 0.26 & 0.86 & 23.59 & & 2.06 & & 4094 & 33.86 & -19.27 & & & & $\mathrm{c}$ & \\
\hline 71868 & 3.1 & 1.28 & 0.17 & -0.52 & 0.42 & 22.97 & 235.32 & & 2.07 & 2799 & 33.01 & -20.42 & -1.86 & -0.50 & -0.87 & $\mathrm{~b}, \mathrm{~d}$ & ARP284, VV51 \\
\hline 72128 & 1.0 & 1.11 & 0.10 & -0.03 & 0.66 & 23.86 & 206.58 & & 2.11 & 2892 & 33.09 & -18.77 & -1.22 & 0.11 & & $\mathrm{~d}$ & \\
\hline 72638 & 1.1 & 1.38 & 0.31 & 0.24 & 0.67 & 23.35 & 609.69 & & 2.49 & 4300 & 33.98 & -21.58 & -2.49 & -0.79 & & d & \\
\hline
\end{tabular}

Notes: the presence of the galaxy in Arp or Vorontsov Velyaminov atlases is reported with the original name (ARP and VV). The presence of peculiar kinematics such as counterrotation is indicated with CR. REFERENCES: a: Boselli, A., Gavazzi, G., Lequeux, J., Buat, V., Casoli, F., Dickey, J., \& Donas, J. 1995, A\&A, 300, L13; b: Young, J. S., Xie Shuding, Tacconi, L. J., et al. 1995, ApJS, 98, 219; c: Knapp, G. R., Guhathakurta, P., Kim, D.-W., \& Jura, M. 1989, ApJS, 70, 329; d: (LEDA) Paturel, G., Andernach, H., Bottinelli, L., Di Nella, H., Durand, N., Garnier, R., Gouguenheim, L., Lanoix, P., Martinet, M. C., Petit, C., Rousseau, J., Theureau, G., \& Vauglin, I. 1997, A\&AS, 124, 109; e: Roberts, M., Hogg, D. E., Bregman, J. N., Forman, W. R., \& Jones, C. 1991, ApJS, 75, 751; f: Sage, L. J. 1993, A\&A, 100, 537 and A\&A, 272, 123; g: van Driel, W., Ragaigne, D., Boselli, A., Donas, J., \& Gavazzi, G. 2000, A\&AS, 144, 463; h: Zhu, Ming, Seaquist, E. R., Davoust, Emmanuel, Frayer, David, T., Bushouse, Howard 1999, AJ, 118, 145. 